ACCEPTED MANUSCRIPT

\title{
A piezoelectric beam model with geometric, material and damping nonlinearities for energy harvesting
}

To cite this article before publication: Claudio David Gatti et al 2020 Smart Mater. Struct. in press https://doi.org/10.1088/1361-665X/ab9ddb

\section{Manuscript version: Accepted Manuscript}

Accepted Manuscript is "the version of the article accepted for publication including all changes made as a result of the peer review process, and which may also include the addition to the article by IOP Publishing of a header, an article ID, a cover sheet and/or an 'Accepted

Manuscript' watermark, but excluding any other editing, typesetting or other changes made by IOP Publishing and/or its licensors"

This Accepted Manuscript is @ 2020 IOP Publishing Ltd.

During the embargo period (the 12 month period from the publication of the Version of Record of this article), the Accepted Manuscript is fully protected by copyright and cannot be reused or reposted elsewhere.

As the Version of Record of this article is going to be / has been published on a subscription basis, this Accepted Manuscript is available for reuse under a CC BY-NC-ND 3.0 licence after the 12 month embargo period.

After the embargo period, everyone is permitted to use copy and redistribute this article for non-commercial purposes only, provided that they adhere to all the terms of the licence https://creativecommons.org/licences/by-nc-nd/3.0

Although reasonable endeavours have been taken to obtain all necessary permissions from third parties to include their copyrighted content within this article, their full citation and copyright line may not be present in this Accepted Manuscript version. Before using any content from this article, please refer to the Version of Record on IOPscience once published for full citation and copyright details, as permissions will likely be required. All third party content is fully copyright protected, unless specifically stated otherwise in the figure caption in the Version of Record.

View the article online for updates and enhancements. 


\title{
A piezoelectric beam model with geometric, material and damping nonlinearities for energy harvesting
}

\author{
Claudio D. Gatti1,2, Mariano Febbo ${ }^{3}$, Sebastián P. Machado ${ }^{1,2}$ and Santiago M. Osinaga ${ }^{1,2}$ \\ 1 Consejo Nacional de Investigaciones Científicas y Técnicas (CONICET), Argentina. \\ ${ }^{2}$ Grupo de Investigación en Multifísica Aplicada (GIMAP), Universidad Tecnológica \\ Nacional FRBB, 11 de Abril 461, 8000 Bahía Blanca, Argentina. \\ 3Instituto de Física del Sur (IFISUR) and Departamento de Física, Universidad Nacional del \\ Sur (UNS), CONICET, AvAlem 1253, 8000 Bahía Blanca, Argentina
}

\begin{abstract}
To predict electrical generation in piezoelectric small-scale beam energy harvesting devices, it is important to have a complete mathematical model that captures the different associated phenomena. In the literature, some authors propose several alternatives of nonlinear mathematical formulations, with non-linearities coming from different physical aspects. All these formulations present good aptitudes to predict the nonlinear behavior of the system under different values of accelerations, geometry and boundary conditions. At the same time, they do not represent a unified general proposal for modeling multimodal energy harvesting devices of any type of mode generation and boundary conditions at large excitations. In this sense, this paper presents a mathematical description of inextensional nonlinear Euler-Bernoulli piezoelectric beams that combines the best contributions of the literature to the voltage generation of multimodal nonlinear piezoelectric energy harvesters (geometric, material and damping non-linearities). The developed analytical model yields a total set of $N+1$ ordinary differential equations for the first $N$ modes and for the output voltage. However, direct solution of this ordinary nonlinear differential system of $N$ equations is computationally costly. Instead, a reduced algebraic system of $2(N+1)$ algebraic equations is proposed applying the method of averaging. Its main advantage is that it makes more suitable and computationally economical for the implementation of a parameter identification process involving any number of piezoelectric inserts (unimorph or bimorph) and mode of generation $\left(\mathrm{d}_{33}\right.$ or $\left.\mathrm{d}_{31}\right)$. Two types of validations are presented for some selected physical systems to test the validity of the assumptions: a numerical one, by the direct integration of the equations of motion and an experimental one. A final comparison between the results demonstrates the importance of the having a unified nonlinear model to predict the generated voltage in multimodal energy harvesters.
\end{abstract}


Keywords: Piezoelectric Energy Harvesting; Material, Geometrical and Damping Nonlinearities; Multimodal systems; Reduced algebraic equations.

* Corresponding author; e-mail: mfebbo@uns.edu.ar.

\section{INTRODUCTION}

Energy harvesting is one of the fundamental issues in the technological application of small-scale energy sources for the industry.

In particular, the harvest of energy based on the deformation of a piezoelectric material [1]caused by the vibration of the motors of the transport vehicles, is of interest in this work. Various types of devices have been reported in the literature for this purpose, from simple cantilever beams [2] to sophisticated multimodal devices [3, 4]. However, none of them seems to be definitive in the technological application of these types of devices.

Regarding the mathematical formulations used to model micro-power generation systems, many researchers have developed linear models of energy harvesting devices such as Beeby et al. [5] and Erturk et al. [6]. These models mostly use the theory of BernoulliEuler for the structural beam elements under base acceleration, considering a linear constitutive piezoelectric equation and Rayleigh proportional damping [7]. However, these formulations are accurate only for low excitation amplitudes [6]. As the amplitude of the base acceleration increases, linear models overestimate the electrical generation in a resonant condition, due to the intrinsic non-linearity of piezoelectric materials [8]. For this reason, nonlinear models are essential to describe the dynamic behavior of these systems and to predict energy generation in piezoelectric energy harvesters, as considered by different authors.

Several analytical approaches have been applied to study this problem. Between them, some authors present their models considering only geometric nonlinearities due to large displacements [9]. Other authors, instead, focus their studies modeling material nonlinearities such as the nonlinear elastic behavior, the nonlinear electromechanical coupling or ferroelastic and ferroelectric hysteresis [10-17]. Generally speaking, the addition or not of these nonlinear phenomena in the constitutive equations will depend on the strength of the electric field or the applied stress.

Concerning the proposals of non-linear constitutive equations, Joshi [10] presented in 1992 non-linear constitutive relations for piezoceramic materials, which would later be the basis of many works on applied piezoelectricity. He deduced the constitutive equations from a thermodynamic viewpoint based on Gibbs free energy. His nonlinear second order relations include non-linear elasticity terms, non-linear elasto and electric-striction terms and non-linear permittivity effects. Later in 2006, Bertotti and Mayergoyz by one side [11] and Damjanovicby the other side [12] presented in two textbooks a meticulous study on the hysteresis phenomenon in piezoelectric and ferroelectric materials. Following a similar approach, Goldschmidtboeing et al. [13] analyzed the influence of ferroelastic hysteresis on cantilever beams of PZT material, mechanically excited at the base. On the other hand, Stanton et al. [8] experimentally validated a mathematical model taking into account the non-linearity corresponding to high-order elastic effects and non-linear coupling associated with an energy harvesting circuit. Aurelle et al. [14] studied the contribution of deformations and electromechanical coupling in the non-linear response of a piezoelectric beam under weak electric fields in order to focus their study on nonlinearities separately. Following this line, Albareda et al. [15] considered a high-order formulation in the thermodynamic potential from which non-linear constitutive equations for the voltage and high-order electrical displacement in the deformation and electric field are obtained. Priya et al. [16] analyzed electrical non-linearities generated by strong electric fields and the influence of the ferroelastic phenomenon. Leadenham and Erturk [17] studied a model of 
distributed parameters taking into account the softening effect and dissipative nonlinearities caused by ferroelastic hysteresis of a bimorph piezoelectric beam of PZT 5A connected in series in its first flexional mode using the method of averaging. This formulation for the piezoelectric material was experimentally validated, both in actuation and in energy harvesting applications.

The need to unify these non-linear models in a single work that combines the best characteristics of previous contributions for a general multimodal system constitutes the aim of this paper. In this sense, this work proposes a generalized and unified non-linear mathematical model that combines the quadratic non-linear viscous dissipation proposed by Stanton et al. [8], the non-linear constitutive model proposed by Leadenham and Erturk [17] and a geometric non-linearity due to large deformations such as the one reported by Mak et al. [9]. In summary, the proposed model is capable to capture the physical phenomena that are observed in piezoelectric materials for energy harvesting due to large excitation amplitudes by the modification of its geometric, dissipative, structural and electrical characteristics. This includes the analytical description of $d_{31}$ or $d_{33}$ piezoelectric modes and unimorph or bimorph configurations in a beam-type structural element using an arbitrary number of structural modes for any type of boundary conditions. Additionally, the system of $N+1$ differential equations obtained from the model is reduced to a $2(N+$ 1) generalized system of algebraic equations by means of the method of averaging. The numerical and experimental validation of the proposal is also presented for three different cases using a Macro Fiber Composite (MFC) piezoelectric sheet.

The paper is organized as follows. A detailed mathematical treatment of a multimodal device [18] consisting of a composite beam (piezoelectric + substructure) with two massspring systems attached to their ends which serves as a general application of the proposed formulation is presented in section 2 .

Section 3 presents the reduction of the system of ordinary differential equations to a system of algebraic equations, using the method of averaging [19]. The obtained system is validated for electromechanical devices under any type of piezoelectric inserts and their physical configurations (unimorph or bimorph). Finally, section 4 presents the reduced system which is computationally and experimentally validated for a cantilever system in $d_{31}$ and $d_{33}$ mode generation as well as for the proposed multimodal system.

Concluding remarks summarize the main findings of the present proposal at the end of the paper.

\section{THEORETICAL MODEL AND ELECTROMECHANICAL EQUATIONS}

In this section we present a detailed non-linear formulation of a multimodal energy harvesting device (MEHD) previously presented by the authors in [20] using a linear theory. Despite of this fact, the final general equations can be applied for any mode of generation $\left(\mathrm{d}_{31}\right.$ or $\left.\mathrm{d}_{33}\right)$ and physical configuration (unimorph-bimorph).

Figure1 shows the MEHD comprising a composite beam with two spring-mass systems at the ends of it. The natural frequencies of the device can be chosen by a proper selection of system's parameters. The composite beam is represented by a substructure (steel or aluminum) and a sheet of piezoelectric material on the upper surface of the substructure (unimorph configuration). After analyzing different commercially available options, a PZT$5 \mathrm{~A}$ macro fiber composite (MFC) with interdigitated electrodes (manufactured by SmartMaterials, Inc) is used in the present study, since it presents important advantages over those conventionally used $[20,21]$.

In Figure $1, w(x, t)$ is the relative vertical deflection of the beam, $g(t)$ is the temporary excitation of the base, $L$ is the total length of the composite beam and $m_{1,2}, k_{1,2}$ and $k_{t 1,2}$ are 
the masses and the vertical and torsional stiffness of the springs at the ends $x=0$ and $x=$ $L$ respectively. In addition, $a, b_{p}, h_{p}, b_{s}$ and $h_{s}$ are the distance from the bottom piezoelectric side to the neutral fiber, the width and thickness of the piezoelectric sheet, and the width and thickness of the substructure, respectively.
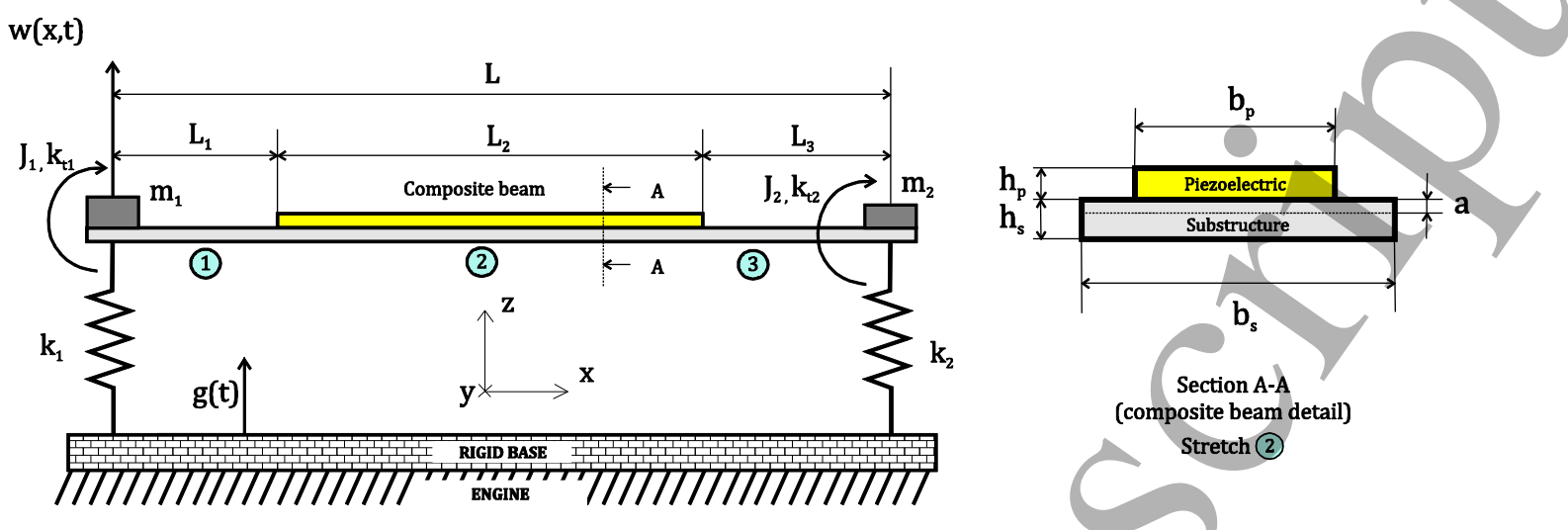

Figure1:Schematics of the model (left) with a detailed view of the composite beam (right).

On the other hand, Figure 2 shows the basic electrical circuit that provides the voltage $v(t)$ generated by the device. There, it is possible to observe the electric model of the piezoelectric considered as a current generator $i(t)$, with an internal ideal capacitor $C_{p}$ and a load resistance $R_{l}$ connected in parallel.

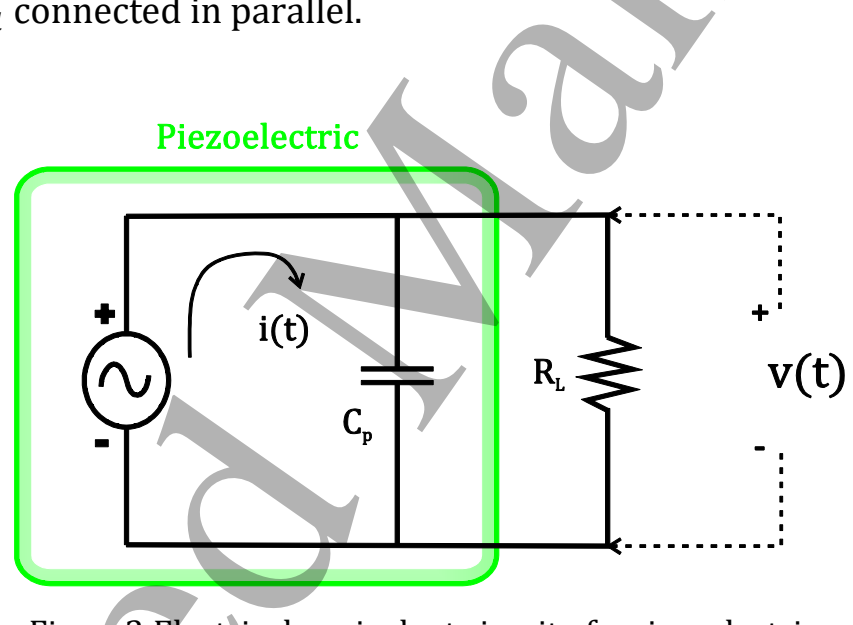

Figure2:Electrical equivalent circuit of a piezoelectric.

\subsection{ELECTROMECHANICAL MODEL OF THE SYSTEM}

The piezoelectric beam is modeled according to the Euler-Bernoulli formulation [22], considering only the vertical displacement and assuming that it is inextensible. This last assumption implies, for example, that it can not be applied for beams subject to large centrifugal forces, beams with fixed boundary conditions, etc [23].

A Lagrangian approach [24] is used to build up the system of differential equations. To consider the material nonlinearity, a similar model such as the one presented by Leadenham and Erturk [17] which considers a non-linear elasticity coefficient $\left(c_{111}^{p}\right)$ and a non-linear coupling coefficient given by a non-linear piezoelectric constant $\left(e_{333}\right)$ is used. However, in contrast to [17] a structurally more complex piezoelectric beam with interdigitated electrodes is used to carry out this study. Regarding the dissipation of energy in the MEHD, a viscous non-linear quadratic model is selected, as presented by Stanton et al. [8] for a structurally different type of piezoelectric material. This model demonstrates to have a good agreement with experimental tests in piezoelectric beam-like systems such as the one 
presented in this work.

In the following, the axes $x, y, z$, shown in Figure 1 correspond to the subscripts 1,2 and 3 , respectively. The points in the upper part of the variables $(\cdot, \cdot, \ldots)$ refer to the temporal derivatives, the quotes $\left({ }^{\prime}, \quad, \ldots\right)$ to the derivatives with respect to the coordinate $x$ and the displacement in the $z$ direction is $w(x, t)$. In addition, the superscripts $p$ and $s$ refer to the piezoelectric and substructure, respectively.

The displacement vector, without considering pure axial displacements and incorporating the temporal excitation of the base $g(t)$, is:

$$
\boldsymbol{u}=\left[\begin{array}{lll}
u_{1} & u_{2} & u_{3}
\end{array}\right]^{t}=\left[\begin{array}{lll}
-z w^{\prime} & 0 & w+g(t)
\end{array}\right]^{t}
$$

The axial strain of a differential element is given by [24]

$$
e=\sqrt{\left(1+u_{1}^{\prime}\right)^{2}+u_{3}^{\prime 2}}-1
$$

After considering an inextensible beam $e=0$, it is possible to obtain:

$$
\begin{gathered}
u_{1}^{\prime}=-\frac{1}{2} u_{3}^{\prime 2} \\
u_{1}=-\frac{1}{2} \int_{0}^{l} u_{3}^{\prime 2} d l
\end{gathered}
$$

In this sense, non-linear geometric strains are given by the following local expression:

$$
\varepsilon_{1}=-z\left(w^{\prime \prime}+\frac{1}{2} w^{\prime \prime} w^{\prime 2}\right)
$$

The first term on the right side of (4) is the axial strain of a problem of small displacements due to bending, while the second is the axial strain considering bending at large displacements.

The expression of the enthalpy density of the piezoelectric material $\left(H^{p}\right)$ and of that the substructure $\left(H^{S}\right)$ are defined as [17]:

$$
\begin{gathered}
H^{p}=\frac{1}{2} c_{11}^{p} \varepsilon_{1}{ }^{2}+\frac{1}{3} c_{111}^{p} \varepsilon_{1}{ }^{3} \operatorname{sign}\left(\varepsilon_{1}\right)-e_{33} \varepsilon_{1} E_{1}-\frac{1}{2} e_{333} \varepsilon_{1}{ }^{2} E_{1} \operatorname{sign}\left(\varepsilon_{1}\right) \\
-\frac{1}{2} \epsilon_{11}^{\varepsilon} E_{1}{ }^{2} \\
H^{s}=\frac{1}{2} c_{11}^{S} \varepsilon_{1}{ }^{2}
\end{gathered}
$$

Where $c_{11}^{p, s}$ are the elasticity modulus of the piezoelectric sheet and the substructure respectively, and $\epsilon_{11}^{\varepsilon}$ is the electric permittivity at constant strain. Additionally, $e_{33}=c_{11}^{p} d_{33}$ is the linear piezoelectric stress constant and $E_{1}$ is the longitudinal electric field in the $x$ direction which is given by, $E_{1}=-v(t) / l_{p}[20]$.

Applying the following relations [10]: 


$$
\sigma_{1}^{p}=\frac{\partial H^{p}}{\partial \varepsilon_{1}} \quad, \quad \sigma_{1}^{S}=\frac{\partial H^{s}}{\partial \varepsilon_{1}} \quad, \quad D_{1}=-\frac{\partial H^{p}}{\partial E_{1}}
$$

it is possible to obtain the axial stresses in the piezoelectric element $\left(\sigma_{1}^{p, s}\right)$ and in the substructure which, as functions of the electric displacement $\left(D_{1}\right)$ result:

$$
\begin{gathered}
\sigma_{1}^{p}=c_{11}^{p} \varepsilon_{1}+c_{111}^{p} \varepsilon_{1}{ }^{2} \operatorname{sign}\left(\varepsilon_{1}\right)-e_{33} E_{1}-e_{333} \varepsilon_{1} E_{1} \operatorname{sign}\left(\varepsilon_{1}\right) \\
\sigma_{1}^{S}=c_{11}^{S} \varepsilon_{1} \\
D_{1}=e_{33} \varepsilon_{1}+\frac{1}{2} e_{333} \varepsilon_{1}{ }^{2} \operatorname{sign}\left(\varepsilon_{1}\right)+\epsilon_{11}^{\varepsilon} E_{1}
\end{gathered}
$$

Both expressions satisfy the necessary and sufficient condition [10]:

$$
\frac{\partial \sigma_{1}^{p}}{\partial E_{1}}=-\frac{\partial D_{1}}{\partial \varepsilon_{1}}
$$

It is important to clarify that the sign of the strain is incorporated in the quadratic terms in order to maintain the non-linear effect in both directions of the deformation of the beam.

The total potential energy of the system, neglecting the gravitational potential energy, is the sum of the elastic potential energies of the beam $U_{b}$ and of the springs $U_{k 1,2}$,

$U=U_{b}+U_{k 1}+U_{k 2}$.

The expression for the beam is given by [6]:

$$
U_{b}=\int_{V_{s}} H^{s} d V_{s}+\int_{V_{p}} H^{p} d V_{p}
$$

The first and second volume integral in (9) correspond to the elements of the substructure and the piezoelectric, respectively. Using expressions (4) and (5), replacing them in the equation (9) and integrating up to length $L$, we obtain:

$$
\begin{array}{r}
U_{b}=\frac{1}{2} \int_{0}^{L}\left[E I w^{\prime \prime 2}+\frac{1}{3} E I_{n} w^{\prime \prime 3} \operatorname{sign}\left(w^{\prime \prime}\right)+E I\left(w^{\prime} w^{\prime \prime}\right)^{2}\right. \\
-2\left(J_{p}(x) w^{\prime \prime}+\frac{1}{2} J_{p n}(x) w^{\prime \prime 2} \operatorname{sign}\left(w^{\prime \prime}\right)\right. \\
\left.\left.+\frac{1}{2} J_{p}(x){w^{\prime}}^{2} w^{\prime \prime}\right) v(t)\right] d x-\frac{1}{2} C_{p} v(t)^{2}
\end{array}
$$

Where $E I$ is the coefficient of linear stiffness which, for the sections 1 and 3 (see Figure 1 ) is $E I_{1,3}$ and for section 2, which contains the piezoelectric sheet, it is $E I_{2}$. In addition, $E I_{n}$ is the coefficient of non-linear stiffness valid only in section 2 due to the non-linear elasticity of the piezoelectric material. The expressions of these coefficients and those mentioned hereinafter are presented in Appendix A.

In view of equation $(10) J_{p}(x)$ is the coefficient of linear electromechanical coupling in section 2 due to the piezoelectric material, and it is defined as: 


$$
J_{p}(x)=J_{p} f_{H}(x)
$$

where $J_{p}$ is the linear electromechanical coupling constant and $f_{H}(x)$ is a function dependent on the generation mode $\left(\begin{array}{lll}\mathrm{d}_{31} & 0 & \mathrm{~d}_{33}\end{array}\right)$ and the piezoelectric type (unimorph or bimorph). This function is introduced to consider the coupling only in the length of the piezoelectric sheet and to retain the corresponding term after the spatial differentiation.

For a $\mathrm{d}_{33}$ generation mode, the electric field is assumed to be uniform in the longitudinal direction $x$ over the effective electrode spacing, as considered in ref [31]. This leads to a linear function for the electric potential (voltage) over the length of the harvester, as can be seen in Figure 12 in the Appendix. Then, the function $f_{H}(x)$ results:

$$
\begin{aligned}
f_{H}(x)=\sum_{i=1}^{n}\{(1 & \left.+\frac{x-x_{i 2}}{x_{i 2}-x_{i 3}}\right)\left[H\left(x-x_{i 2}\right)-H\left(x-x_{i 3}\right)\right] \\
& \left.+\left(\frac{x-x_{i 4}}{x_{i 5}-x_{i 4}}\right)\left[H\left(x-x_{i 4}\right)-H\left(x-x_{i 5}\right)\right]\right\}
\end{aligned}
$$

Where $H(x)$ is the Heaviside function and the $x_{i j}$ can be seen in Figure12 of Appendix A.

For a $d_{31}$ generation mode, the electric field is also assumed to be uniform in the vertical direction $y$, between the electrodes. In this sense, the voltage is constant at the top and bottom electrodes, as can be observed in Figure 14, in the Appendix. Then, the function $f_{H}(x)$ takes the form:

$$
\begin{gathered}
f_{H}(x)=\sum_{i=1}^{n}\left[H\left(x-x_{i 1}\right)-H\left(x-x_{i 1}\right)+H\left(x-x_{i 3}\right)-H\left(x-x_{i 4}\right)+H\left(x-x_{i 5}\right)\right. \\
\left.-H\left(x-x_{i 6}\right)\right]
\end{gathered}
$$

Where $H(x)$ is the Heaviside function and the $x_{i j}$ can be seen in Figure 14 of Appendix A.

In equation (10) $J_{p n}(x)$ is the coefficient of non-linear electromechanical coupling in section 2 , and it is defined as:

$$
J_{p n}(x)=J_{p n} f_{H}(x)
$$

where $J_{p n}$ is the non-linear coupling constant whose approximate expression, considering a constant electric field throughout the length of the piezoelectric, is presented in Appendix A.

To conclude with the potential energy of the beam, it should be clarified that for the nonlinear geometric terms, only the quadratic terms coming from $\varepsilon_{1}{ }^{2}$ were taken into account. In addition, in order to obtain an approximate analytical expression for the sign function, only the component of small displacements of the deformation $\left(\varepsilon_{1}=-z w^{\prime \prime}\right)$ which represent its linear part, is considered for the sign function.

On the other hand, the elastic potential energy of the springs, taking into account the vertical and torsional deformation with respect to the $y$ axis, turns out to be:

$$
U_{k i}=\frac{1}{2}\left[k_{i} w\left(x_{j}, t\right)^{2}+k_{t i} w^{\prime}\left(x_{j}, t\right)^{2}\right], \quad i=1,2, x_{j}=0, L
$$


Similarly, the total kinetic energy of the system is the sum of the kinetic energies of the beam $T_{b}$ and the tip masses at the ends of the beams $T_{m 1,2}$.

Then, $T=T_{b}+T_{m 1}+T_{m 2}$. It should be noted that the rotational inertia of the beam is neglected. However, the rotational inertia of the tip masses is taken into account. Finally, the expression for the beam results [6]:

$$
T_{b}=\frac{1}{2}\left(\int_{V_{s}} \rho_{s}(\dot{w}+\dot{g})^{2} d V_{s}+\int_{V_{p}} \rho_{p}(\dot{w}+\dot{g})^{2} d V_{p}\right)
$$

where $\rho_{p, s}$ is the density of the piezoelectric and the substructure, respectively. Expanding equation (15) and integrating for a length $L$, we obtain:

$$
T_{b}=\frac{1}{2} \int_{0}^{L}\left(\rho A \dot{w}^{2}+2 \rho A \dot{w} \dot{g}\right) d x+\frac{1}{2} m_{t} \dot{g}^{2}
$$

Where $\rho A$ is the unitary mass of the beam, given by: $\rho A_{1,3}$ for sections 1 and 3 , and $\rho A_{2}$ for section 2 . In the last term of equation (16), $m_{t}$ is the total mass of the beam.

The kinetic energy of the masses, taking into account the vertical displacement and their rotational inertias results:

$$
T_{m i}=\frac{1}{2}\left\{m_{i}\left[\dot{w}\left(x_{j}, t\right)+\dot{g}\right]^{2}+J_{i} \dot{w}^{\prime}\left(x_{j}, t\right)^{2}\right\}, i=1,2, x_{j}=0, L
$$

As already mentioned above, for the dissipation of energy in the device, two types of effects are considered: (a) viscous damping in the composite beam and (b) dissipation by Joule effect [25] in the load resistance. For the viscous damping, a linear and a nonlinear quadratic model is proposed, following Stanton [8], Bandstra [26] and Yang [27] adopting the form:

$$
F_{v}=c \dot{w}+c_{n} \dot{w}^{2} \operatorname{sign}(\dot{w})
$$

where $c$ and $c_{n}$ are the linear and non-linear damping coefficients respectively.

In order to apply Hamilton's principle for deducing the electromechanical equations, we include these dissipation effects via the work of non-conservative forces $\delta W_{n c}$ resulting in:

$$
\delta W_{n c}=-F_{v} \delta w-i \delta \lambda
$$

Where $\lambda$ is the electric flow and the electric current $i$ is calculated by Ohm's Law as $i=\dot{\lambda} / R_{l}$ where $\dot{\lambda}=v$ is the voltage in the load resistance.

\subsection{Electromechanical Lagrange Equations}

Applying Hamilton's principle we obtain the Lagrange equations [24] for the system considered: 


$$
\begin{gathered}
\frac{\partial}{\partial t}\left(\frac{\partial L}{\partial \dot{w}}\right)+\frac{\partial}{\partial x}\left(\frac{\partial L}{\partial w^{\prime}}\right)-\frac{\partial}{\partial x^{2}}\left(\frac{\partial L}{\partial w^{\prime \prime}}\right)+c \dot{w}+c_{n} \dot{w}^{2} \operatorname{sign}(\dot{w})=0 \\
\frac{\partial}{\partial t}\left(\frac{\partial L}{\partial v}\right)+\frac{v}{R_{l}}=0
\end{gathered}
$$

where the Lagrangian is $L=T-U$.

Then equation (20) becomes:

$$
\begin{aligned}
\rho A \ddot{w}+c \dot{w}+c_{n} \dot{w}^{2} \operatorname{sign}(\dot{w})+E I w^{I V}+E I_{n}\left(w^{\prime \prime \prime 2}+w^{\prime \prime} w^{I V}\right) \operatorname{sign}\left(w^{\prime \prime}\right) \\
+E I\left(w^{\prime \prime 3}+4 w^{\prime} w^{\prime \prime} w^{\prime \prime \prime}+w^{\prime 2} w^{I V}\right)-J_{p}^{\prime \prime} v \\
-v J_{p n} w^{I V} \operatorname{sign}\left(w^{\prime \prime}\right)-v\left(J_{p}^{\prime} w^{\prime} w^{\prime \prime}+\frac{1}{2} J_{p}^{\prime \prime} w^{\prime 2}\right)=-\rho A \ddot{g}
\end{aligned}
$$

and equation (21) turns into:

$$
\begin{gathered}
C_{p} v+\frac{v}{R_{l}}+\int_{0}^{l}\left[\left(J_{p}+J_{p n} w^{\prime \prime} \operatorname{sign}\left(w^{\prime \prime}\right)+\frac{1}{2} J_{p} w^{\prime 2}\right) \dot{w}^{\prime \prime}+J_{p} w^{\prime} w^{\prime \prime} \dot{w}^{\prime}\right] d x \\
=0
\end{gathered}
$$

These expressions are a generalization of others previously proposed in the literature, particularly for the nonlinear terms. The equation proposed by Leadenham and Erturk [17] can be recovered by neglecting the nonlinear geometrical terms (sixth term of the left hand side in Eq. 22 and fifth and sixth terms of the left hand side in Eq. 23) and modifying the damping terms (second and third terms of the left hand side in Eq. 22) by his own proposal. Additionally, there is a difference in the last term of the left hand side in Eq. 22 due to $f_{H}(x)$, which depends on the interdigitated electrodes of the MFC (Leadenham and Erturk [17] used a piezo sheet T226-A4-103X with no fibers). On the other hand, the equations proposed by Stanton et al. [8] can be obtained by eliminating the sign function and the nonlinear geometrical terms in Eqs. 22 and 23.

\subsection{Spatial discretization of electromechanical equations}

One of the main procedures to obtain an analytical solution of the Lagrange equations for continuous media is the spatial discretization of the displacement, applying the classical method of modal expansion by separation of variables. In this method, the deflection of the beam is represented as a finite sum of $N$ generalized coordinates $q_{i}(t)$ multiplied by modal shape functions $\phi_{i}(x)$ as follows:

$$
w(x, t)=\sum_{i=1}^{N} \phi_{i}(x) q_{i}(t)
$$

Modal shape beam-type functions are proposed of the following form:

$$
\phi_{i}(x)=C_{1} \cos \left(\beta_{i} x\right)+C_{2} \cosh \left(\beta_{i} x\right)+C_{3} \sin \left(\beta_{i} x\right)+C_{4} \sinh \left(\beta_{i} x\right)
$$

where the eigenvalues $\beta_{i}$ are related to their respective natural frequencies $\omega_{i}$ by $\omega_{i}=$ $\left(\beta_{i} L\right)^{2} \sqrt{E I / \rho A L^{4}}$ and the constants $C_{j}(j=1-4)$ are determined by the boundary 
conditions and normal mode normalization condition [18].

Replacing equation (24) in (22), multiplying both members by $\phi_{i}(x)$ and integrating in the total length $L$, we obtain a set of $i=1 . . N$ equations of the form:

$$
\begin{aligned}
M_{i} \ddot{q}_{i}+C_{i} \dot{q}_{i}+ & \sum_{j, k=1}^{N} C_{n i j k} \dot{q}_{j} \dot{q}_{k} \operatorname{sign}\left(\dot{q}_{j}\right)+K_{i} q_{i}+\sum_{j, k=1}^{N} K_{n i j k} q_{j} q_{k} \operatorname{sign}\left(q_{j}\right) \\
& +\sum_{j, k, l=1}^{N} K_{G i j k l} q_{j} q_{k} q_{l} \\
& -\left(\theta_{i}+\sum_{j=1}^{N} \theta_{n i j} q_{j} \operatorname{sign}\left(q_{j}\right)+\sum_{j, k=1}^{N} \theta_{G i j k} q_{j} q_{k}\right) \\
& =-M_{a i} \ddot{g}
\end{aligned}
$$

Similarly, using (24) in (23), an electromechanical equation for the voltage $v$ can be obtained:

$$
C_{p} \dot{v}+\frac{v}{R_{l}}+\sum_{i=1}^{N}\left(\psi_{i}+\sum_{j=1}^{N} \psi_{n i j} q_{j} \operatorname{sign}\left(q_{j}\right)+\sum_{j, k=1}^{N} \psi_{G i j k} q_{j} q_{k}\right) \dot{q}_{i}=0
$$

Equation (26) and (27) are the governing electromechanical equations of the energy harvesting model. It is important to note that the terms involving the electromechanical couplings $\psi$ and $\theta$ are different because in this case the electrodes of the piezoelectric sheet do not cover the entire length of the substructure. This situation differs from most works in the literature where the electrode covers the entire surface of the piezoelectric or where this approach is considered valid as an approximation to the real problem.

\section{REDUCTION OF EQUATIONS}

The system of differential equations (26)-(27) is constituted by $N+1$ differential equations, where $N$ is the number of modes. The direct integration of these equations via a numerical method demands an increasingly large time as the number of modes increases, making the problem hard to solve. To overcome this drawback, in this section we propose to reduce the entire non-linear model to a system of algebraic equations by applying the averaging method [19]. The resulting system consists of two subsystems of $N$ mechanical equations, plus two coupled electrical equations, constituting a system of $2(N+1)$ algebraic equations which is computationally economical to solve. An additional advantage lies in the fact that it is easier and faster to apply methods of identification of parameters and optimization methods to improve power generation. However, the reduction is valid for problems in absence of internal resonances between modes. Despite of this drawback, the multimodal character of the reduced solution permits to consider the contribution of $N$ modes to problem, unlike a large body of research work conducted to date $[13,14,17]$.

The averaging method has been widely used to analyze periodic solutions of ordinary non-linear differential equations. A solution is assumed in sines and cosines, replacing the ordinary differential equations by algebraic equations. Then, the error in the approximate solution is minimized with the Galerkin method [29]. Finally, the resulting system of algebraic equations is solved iteratively, with methods such as Newton-Raphson. 
To perform the reduction of equations (26)-(27) applying this method, we first propose a harmonic base acceleration of the form $\ddot{g}(t)=G \cos (\Omega t)$ and then assume that the temporal response of the mode $i, q_{i}^{*}(t)$ and of the voltage $v(t)$, is harmonic of the form:

$$
\begin{gathered}
q_{i}^{*}(t)=q_{i} \cos \left(\Omega t+\phi_{i}\right) \\
v(t)=V \cos (\Omega t+\phi)
\end{gathered}
$$

These equations can be expressed in an equivalent way by means of the following trigonometric relations:

$$
\begin{gathered}
q_{i} \cos \left(\Omega t+\phi_{i}\right)=Q_{(2 i-1)} \cos (\Omega t)+Q_{(2 i)} \sin (\Omega t) \\
V \cos (\Omega t+\phi)=V_{1} \cos (\Omega t)+V_{2} \sin (\Omega t)
\end{gathered}
$$

where

$$
\cos \left(\phi_{i}\right)=\frac{Q_{(2 i-1)}}{q_{i}}, \sin \left(\phi_{i}\right)=\frac{Q_{(2 i)}}{q_{i}}, \cos (\phi)=\frac{V_{1}}{V}, \sin (\phi)=\frac{V_{2}}{V}
$$

from which the displacement modal amplitudes $q_{i}$ and voltage $V$ can be defined as:

$$
\begin{gathered}
q_{i}=\sqrt{Q_{(2 i-1)}^{2}+Q_{(2 i)}^{2}} \\
V=\sqrt{V_{1}^{2}+V_{2}^{2}}
\end{gathered}
$$

Substituting equations (28) and the expression for $\ddot{g}(t)$ into equations (26)-(27) and applying the averaging method yields the following set of $2(N+1)$ algebraic equations in $Q_{(2 i-1)}, Q_{(2 i)}, V_{1}, V_{2}$ :

$$
\begin{aligned}
& +\frac{8}{3 \pi} K_{n}^{*} Q_{(2 i-1)} \\
& +\frac{4}{3 \pi} \theta_{n}^{*}\left[\left(2 Q_{(2 i-1)}^{2}+Q_{(2 i)}^{2}\right) V_{1}+Q_{(2 i-1)} Q_{(2 i)} V_{2}\right] \\
& +\frac{3}{4} K_{G}^{*} Q_{(2 i-1)} \\
& +\frac{1}{4} \theta_{G}^{*}\left[V_{1}\left(3 Q_{(2 i-1)}^{2}+Q_{(2 i)}^{2}\right)+2 Q_{(2 i-1)} Q_{(2 i)} V_{2}\right]+F_{i} \\
& =
\end{aligned}
$$




$$
\begin{aligned}
-M_{i} \Omega^{2} Q_{(2 i)}- & C_{i} \Omega Q_{(2 i-1)}+K_{i} Q_{(2 i)}-\theta_{i} V_{2}-\frac{8}{3 \pi} C_{n}^{*} \Omega^{2} Q_{(2 i-1)} \\
& +\frac{8}{3 \pi} K_{n}^{*} Q_{(2 i)} \\
& +\frac{4}{3 \pi} \theta_{n}^{*}\left[\left(Q_{(2 i-1)}^{2}+2 Q_{(2 i)}^{2}\right) V_{2}+Q_{(2 i-1)} Q_{(2 i)} V_{1}\right] \\
& +\frac{3}{4} K_{G}^{*} Q_{(2 i)} \\
& +\frac{1}{4} \theta_{G}^{*}\left[V_{2}\left(Q_{(2 i-1)}^{2}+3 Q_{(2 i)}^{2}\right)+2 Q_{(2 i-1)} Q_{(2 i)} V_{1}\right]=0 \\
C_{p} \Omega V_{2} & +\frac{V_{1}}{R_{l}}+\Omega \sum_{i=1}^{N} Q_{(2 i)}\left[\psi_{i}+\frac{4}{3 \pi} \psi_{n}^{*}+\frac{1}{4} \psi_{G}^{*}\right]=0 \\
C_{p} \Omega V_{1}- & \frac{V_{2}}{R_{l}}+\Omega \sum_{i=1}^{N} Q_{(2 i-1)}\left[\psi_{i}+\frac{4}{3 \pi} \psi_{n}^{*}+\frac{1}{4} \psi_{G}^{*}\right]=0
\end{aligned}
$$

which constitute the reduced system of differential equations that model the behavior of flexural Euler-Bernoulli beams with electromechanical (piezoelectric) inserts in any type of generation mode ( $\mathrm{d} 31 \mathrm{od} 33$ ) and piezoelectric configuration (unimorph or bimorph). In the previous equations, the following coefficients are defined:

$$
\begin{gathered}
C_{n}^{*}=\frac{q_{1}}{q_{i}} \sum_{j, k=1}^{N} A_{j k} C_{n i j k}, \quad K_{n}^{*}=\frac{q_{1}}{q_{i}} \sum_{j, k=1}^{N} A_{j k} K_{n i j k}, \quad K_{G}^{*}=\frac{q_{1}}{q_{i}} \sum_{j, k, l=1}^{N} q_{j} A_{k l} K_{G i j k l} \\
\theta_{n}^{*}=\frac{q_{1}}{q_{i}^{3}} \sum_{j=1}^{N} A_{i j} \theta_{n i j}, \theta_{G}^{*}=\frac{q_{1}}{q_{i}^{2}} \sum_{j, k=1}^{N} A_{j k} \theta_{G i j k}, \quad \psi_{n}^{*}=\sum_{j=1}^{N} q_{j} \psi_{n i j}, \quad \psi_{G}^{*}=q_{1} \sum_{j, k=1}^{N} A_{j k} \psi_{G i j k}
\end{gathered}
$$

where $A_{i j}=A_{j k}=A_{k l}$ are the components of the so-defined amplitude coefficient matrix $\boldsymbol{A}$, which is expressed as:

$$
\boldsymbol{A}=\left[\begin{array}{ccccc}
\frac{q_{1}^{2}}{q_{1}} & \frac{q_{1} q_{2}}{q_{1}} & \ldots & \ldots & \frac{q_{1} q_{N}}{q_{1}} \\
& \ddots & & & \frac{q_{2} q_{N}}{q_{1}} \\
& & \ddots & & \vdots \\
& & & \ddots & \vdots \\
\text { Sym } & & & & \frac{q_{N}^{2}}{q_{1}}
\end{array}\right]
$$

\section{NumERICAL AND EXPERIMENTAL VALIDATION}

In order to validate the results presented in section (3), here we compare the solutions obtained by the reduced system, equations (32)-(35), to those obtained by direct integration of the equations of motion (Eqs. 22-23) and to experimental results. This is 
carried out for the following cases: a cantilever beam in $\mathrm{d}_{33}$ and $\mathrm{d}_{31}$ generation mode and the multimodal device presented in section 2 . For the numerical validation, the reduced system is solved iteratively using the method of least squares by means of the trust-interval algorithm (trust-region) implemented by Matlab ${ }^{\mathrm{R}}$ solve command. On the other hand, the system of differential equations is integrated numerically through the implementation of an explicit Runge-Kutta formula by the variable step algorithm of Dormand-Prince [30], through the command ode 45 of Matlab ${ }^{\mathrm{R}}$. For the experimental measurements, the systems are excited by its base through an electrodynamical shaker with variable frequency, waveforms and acceleration levels, and the voltage and accelerations are acquired via PCB accelerometers (model 80C) and a NI data acquisition system (model 9230) at a rate of 2048 samples/sec.

\subsection{CANTILEVER $\mathbf{d}_{33}$ BEAM}

The cantilever beam of Figure 3 is selected for the first case experimental and numerical validation of the reduced equations. A piezoelectric sheet MFC 8507-P1 bonded over a steel beam constitutes the piezoelectric system which generates in $d_{33}$ mode.

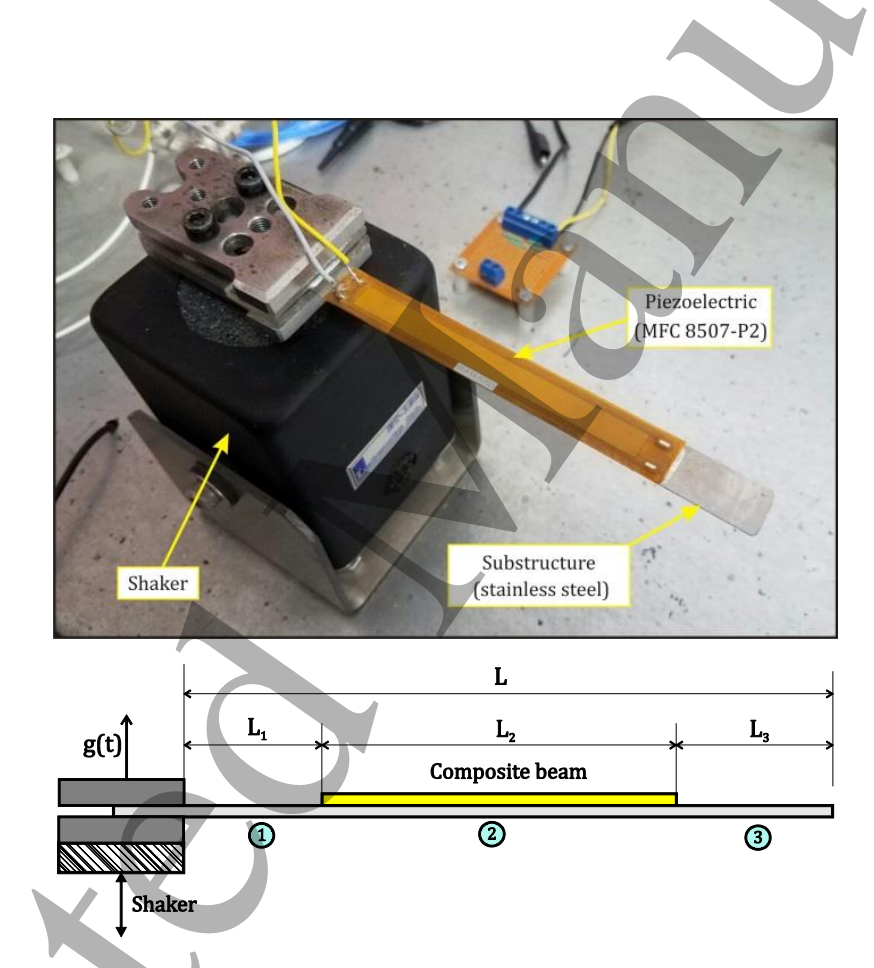

Figure 3: Schematic representation of the cantilever model and experimental setup.

Numerical values of the physical parameters of the cantilever system can be found in Table 1.

To obtain the linear and nonlinear coefficients that were introduced in the analytical model, we carry out an identification process. This process was performed in two stages, minimizing the norm of the difference between theoretical and experimental data over a region that includes the first mode of a cantilever $d_{33}$ beam. The first stage includes the identification of the linear coefficients at very low (constant) acceleration amplitude of 0.04 $\mathrm{g}\left(\mathrm{g}=9.8 \mathrm{~m} / \mathrm{s}^{2}\right)$ with an electrical load of $255 \mathrm{k} \Omega$. As a result, the linear coefficient $\xi_{1}$ and the correction factors $\alpha$ (included in the definition of matrices $\theta, \theta_{\mathrm{n}}, \theta_{\mathrm{G}}$ ) and $\beta$ (included in $\psi$, $\psi_{\mathrm{n}} \mathrm{y} \psi_{\mathrm{G}}$ ) were simultaneously identified. 
The second stage devotes to identify the nonlinear coefficients. To this end, the numerical values of linear coefficients identified in the first stage were introduced into the model. Then, we performed the identification process at $1 \mathrm{~g}$ of constant acceleration amplitude with an electrical load of $255 \mathrm{k} \Omega$. As a result, the nonlinear coefficients $\xi_{\mathrm{n} 1}, \mathrm{c}_{111}^{\mathrm{p}}$ and $\mathrm{e}_{333}$ were simultaneously identified.

Several tests were performed at constant base acceleration with values of $1,0.5,0.1$ and $0.04 \mathrm{G}$, while measuring the stationary voltage in the range of $14-26 \mathrm{~Hz}$. Figure 4 shows the results for: the experimental cases (circles), the reduced model considering only one mode of vibration (Eqs. 32-34 for $N=1$, solid lines) and the curves obtained by direct integration of the equations of motion (Eqs. 26-27) for $N=1$ and $N=3$, which are plotted as dashed lines and dash-dotted lines, respectively.

\begin{tabular}{cccc}
\hline Coefficient & Value & Coefficient & Value \\
\hline$L_{1}$ & $17.6 \mathrm{~mm}$ & $\rho_{s}$ & $7900 \mathrm{~kg} / \mathrm{m}^{3}$ \\
$L_{2}$ & $85 \mathrm{~mm}$ & $\rho_{p}$ & $7750 \mathrm{~kg} / \mathrm{m}^{3}$ \\
$L_{3}$ & $21.8 \mathrm{~mm}$ & $c_{11}^{s}$ & $193 \mathrm{GPa}$ \\
$L$ & $124.4 \mathrm{~mm}$ & $c_{11}^{p}$ & $61 \mathrm{GPa}$ \\
$a$ & $0.176 \mathrm{~mm}$ & $c_{111}^{p}$ & 0 \\
$h_{s}$ & $0.38 \mathrm{~mm}$ & $d_{33}$ & $440 \mathrm{pm} / \mathrm{V}$ \\
$b_{s}$ & $12.7 \mathrm{~mm}$ & $e_{333}$ & $6.5663 \times 10^{4}$ \\
$h_{f}$ & $0.18 \mathrm{~mm}$ & $\epsilon_{11}^{\varepsilon}$ & $15.3 \mathrm{nF} / \mathrm{m}$ \\
$b_{f}$ & $0.355 \mathrm{~mm}$ & $C_{p}$ & $1.5 \mathrm{nF}$ \\
$h_{k}$ & $0.06 \mathrm{~mm}$ & $\xi_{1}$ & 0.0115 \\
$l_{p}$ & $0.41 \mathrm{~mm}$ & $\xi_{n 1}$ & $1.387 \times 10^{-4}$ \\
$w_{p}$ & $0.097 \mathrm{~mm}$ & $\alpha$ & 0.2 \\
$n_{f}$ & 18 & $\beta$ & 0.2 \\
\hline
\end{tabular}

Table 1: Material and geometric parameters of the cantilever model ( $\mathrm{d}_{33}$ generation).

From the results it can be observed that both, the reduced system and the direct integration of the ODES present a good agreement between them and, in turn, fit satisfactorily to the experimental values. This implies that the reduced system predicts with good accuracy the output voltage for different acceleration amplitudes, including those cases not considered in the identification process. Regarding the number of modes assumed in the solution, the direct integration of the ODES shows no sensitive difference to this number. It is important to note that, for the maximum value of acceleration $(1 \mathrm{G})$, a hardening nonlinearity is evidenced for this type of physical system. 

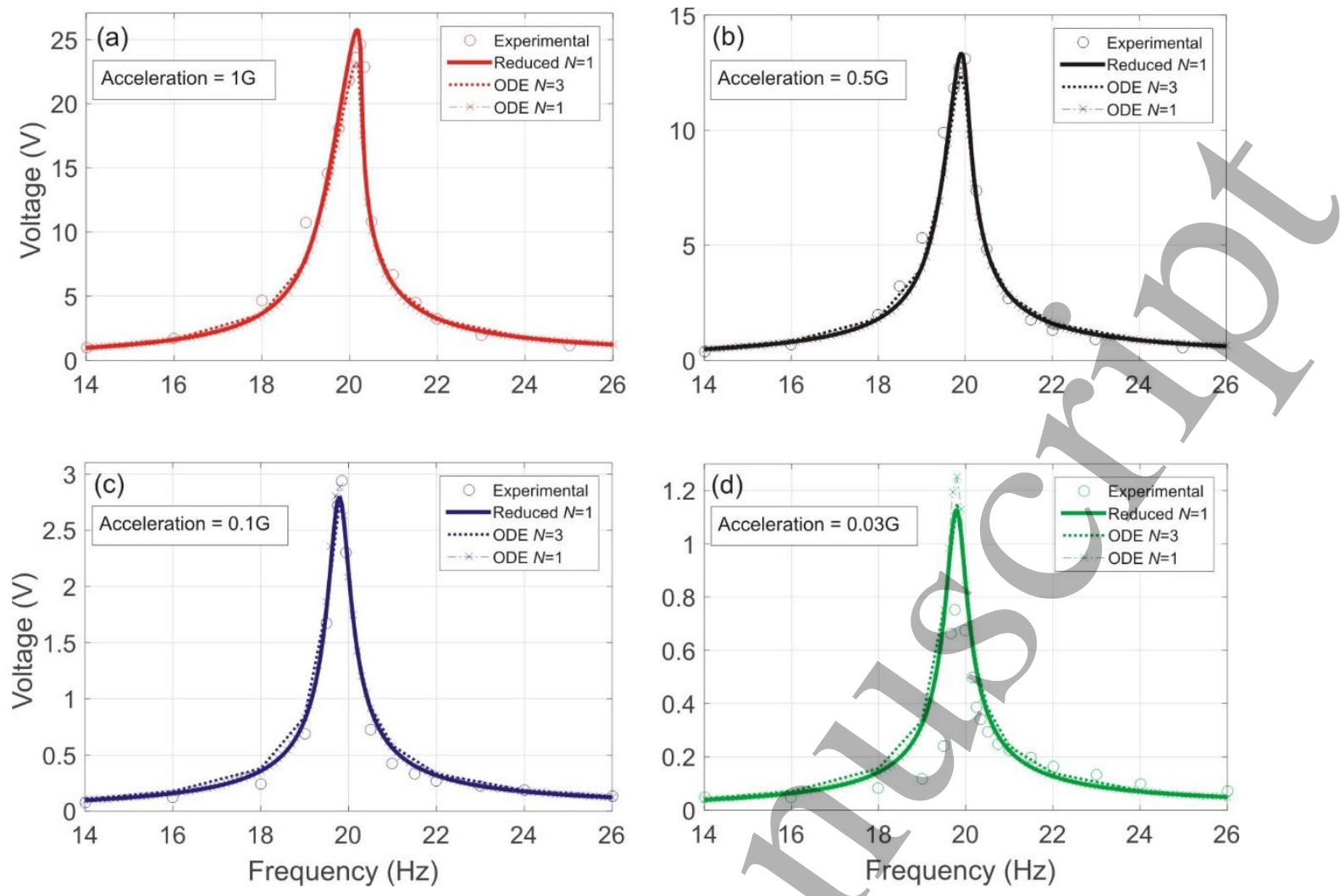

Figure 4: Output voltage for different methods: reduced model with $N=1$ (Reduced $N=1$ ), direct integration of the equations of motion with $N=1$ and $N=3$ (ODE $N=1$ and ODE $N=3$ ), and experimental results for a cantilever beam in $\mathrm{d}_{31}$ generation mode.(a) for $1 \mathrm{~g}$ of base acceleration, (b) for $0.5 \mathrm{~g}$ of base acceleration, (c) for $0.1 \mathrm{~g}$, and (d) for $0.03 \mathrm{~g}\left(\mathrm{~g}=9.8 \mathrm{~m} / \mathrm{s}^{2}\right)$.

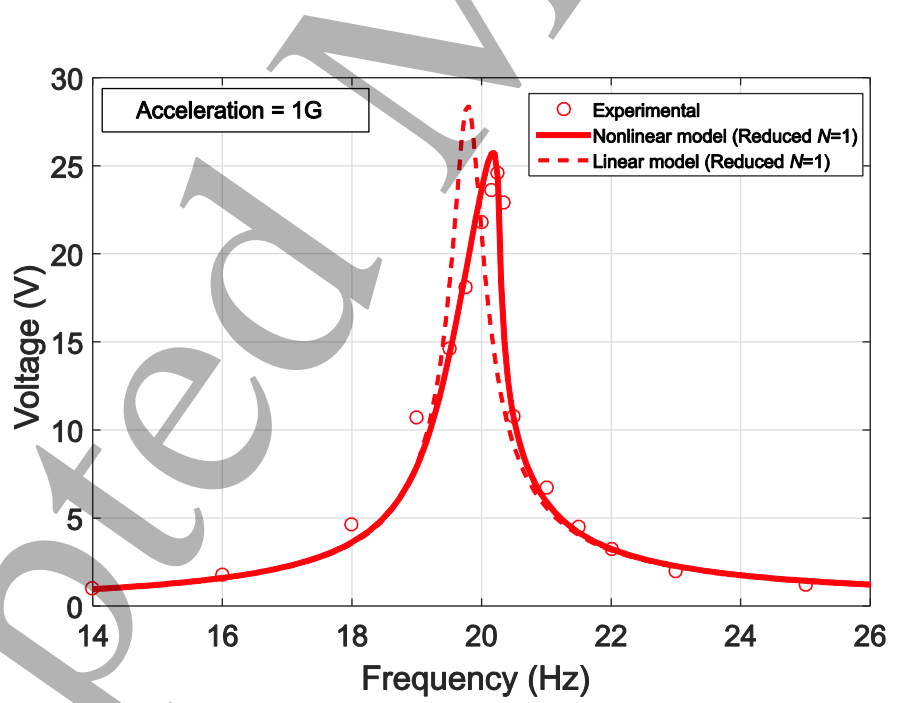

Figure 5: Comparison between linear and nonlinear model in $\mathrm{d}_{33}$ generation mode.

Figure 5 presents the comparison with a linear model which is implemented equating to zero the nonlinear terms of the reduced equations (32)-(35)with $N=1$. From the results, it is possible to observe an overestimation of the generated voltage by an amount of more than 3 Volts, which represents more than $10 \%$ of the predicted value of the nonlinear model.

\subsection{CANTILEVER $\mathbf{d}_{31}$ BEAM}

In a similar manner, a cantilever beam with a piezoelectric sheet MFC 8507-P2 is used to 
build the piezoelectric system which generates in $\mathrm{d}_{31}$ mode.

Table 2 shows the numerical values of the physical parameters of the cantilever system.

Following the same approach presented in section 4.1, the linear and nonlinear coefficients were obtained from an identification process following two stages. In the first stage, the linear parameters $\xi_{1}$ and $\gamma$ were identified at very low (constant) acceleration amplitude of $0.04 \mathrm{~g}$ and electrical load of $255 \mathrm{k} \Omega$. The second stage identified the nonlinear coefficients $\xi_{\mathrm{n} 1}, \mathrm{c}_{111}^{\mathrm{p}}$ and $\mathrm{e}_{311}$. The identification process was carried out at $1 \mathrm{~g}$ of constant acceleration amplitude using the linear parameters obtained in the first stage and the same electrical load of $255 \mathrm{k} \Omega$.

\begin{tabular}{cccc}
\hline Coefficient & Value & Coefficient & Value \\
\hline$L_{1}$ & $17.2 \mathrm{~mm}$ & $c_{11}^{s}$ & $193 \mathrm{GPa}$ \\
$L_{2}$ & $85 \mathrm{~mm}$ & $c_{11}^{p}$ & $61 \mathrm{GPa}$ \\
$L_{3}$ & $21.5 \mathrm{~mm}$ & $c_{111}^{p}$ & $-2.685 \times 10^{12}$ \\
$L$ & $123.7 \mathrm{~mm}$ & $d_{31}$ & $-190 \mathrm{pm} / \mathrm{V}$ \\
$a$ & $0.176 \mathrm{~mm}$ & $e_{311}$ & $9.4553 \times 10^{3}$ \\
$h_{s}$ & $0.38 \mathrm{~mm}$ & $\epsilon_{33}^{\varepsilon}$ & $16.81 \mathrm{nF} / \mathrm{m}$ \\
$h_{p}$ & $0.3 \mathrm{~mm}$ & $C_{p}$ & $49 \mathrm{nF}$ \\
$b_{s}$ & $12.7 \mathrm{~mm}$ & $\xi_{1}$ & 0.0059 \\
$b_{p}$ & $8 \mathrm{~mm}$ & $\xi_{n 1}$ & $3.0071 \times 10^{-4}$ \\
$\rho_{s}$ & $7900 \mathrm{~kg} / \mathrm{m}^{3}$ & $\gamma$ & 0.65 \\
$\rho_{p}$ & $7750 \mathrm{~kg} / \mathrm{m}^{3}$ & & \\
\hline
\end{tabular}

Table 2: Material and geometric parameters of the cantilever model ( $\mathrm{d}_{31}$ generation).

Figure 5 shows the results at constant base acceleration considering 1, 0.5, 0.1 and 0.04 $\mathrm{G}$, while measuring the stationary voltage in the range $14-26 \mathrm{~Hz}$. A very good agreement is observed for all the analyzed cases. This is, the experimental curves (circles), the reduced model curves with one mode $(N=1$, solid lines) and the curves which are the result of the direct integration of the equations of motion with 1 and 3 modes $(N=1$, dashed lines, $N=3$, dash-dotted lines). In contrast with the $\mathrm{d}_{33}$ case, a softening nonlinearity can be observed for the maximum considered acceleration of $1 \mathrm{G}$. Additionally, the generated voltage is larger in comparison with the previous case $\left(\mathrm{d}_{33}\right)$. For example, for a base acceleration of $1 \mathrm{G}$, the maximum voltage is $46 \mathrm{~V}$ for the MFC 8507-P2 $\left(\mathrm{d}_{31}\right)$ and $26 \mathrm{~V}$ for the MFC 8507-P1 $\left(\mathrm{d}_{33}\right)$. This difference is still larger for lower accelerations, for example $0.1 \mathrm{G}$, getting values of 9 and $3 \mathrm{~V}$ for MFC 8507-P2 and MFC 8507- P1, respectively. 

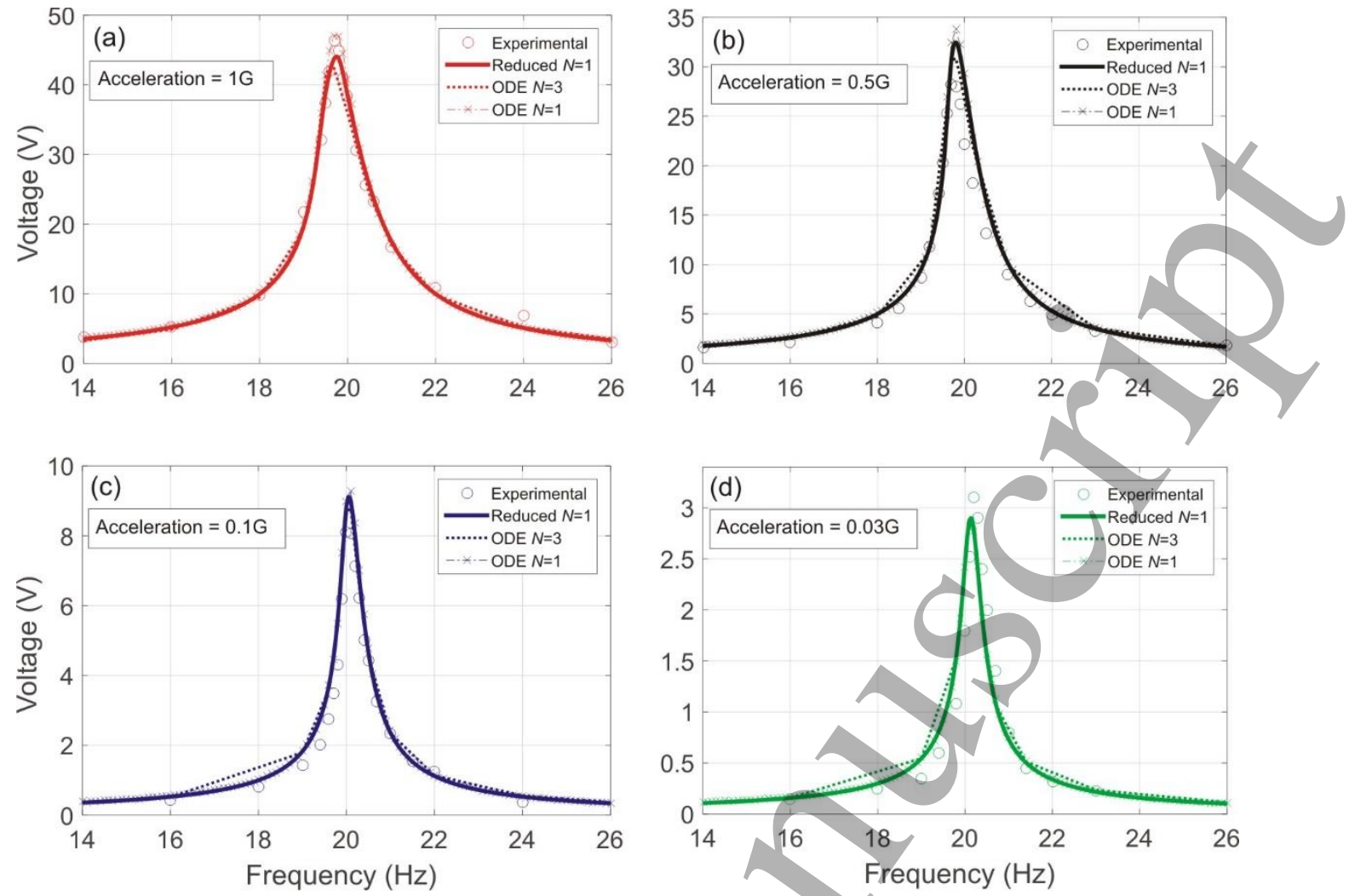

Figure 6: Output voltage for different methods: reduced model with $N=1$ (Reduced $N=1$ ), direct integration of the equations of motion with $N=1$ and $N=3$ (ODE $N=1$, ODE $N=3$ ), and experimental results for a cantilever beam in $\mathrm{d}_{31}$ generation mode. (a) for $1 \mathrm{~g}$ of base acceleration, (b) for $0.5 \mathrm{~g}$ of base acceleration, (c) for $0.1 \mathrm{~g}$, and (d) for $0.03 \mathrm{~g}\left(\mathrm{~g}=9.8 \mathrm{~m} / \mathrm{s}^{2}\right)$

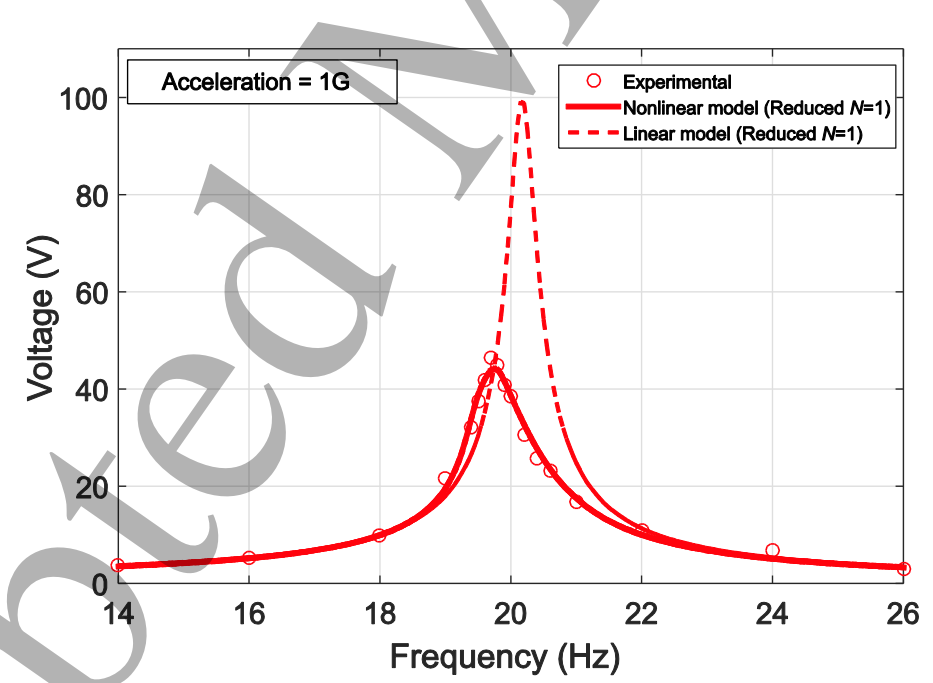

Figure 7: Comparison between linear and nonlinear model in $\mathrm{d}_{31}$ generation mode.

Similarly, Figure 7 presents the comparison with a linear model. This time, the overestimation of the generated voltage is notably larger compared with the $\mathrm{d}_{33}$ case. It is possible to observe a difference of more than a $100 \%$ between the nonlinear predictions, the experiments and the linear values for an acceleration of $1 \mathrm{G}$.

Figure 8 presents the contribution to the voltage of the nonlinear terms of equations (26)(27) in $\mathrm{d}_{31}$ generation mode. The different terms are labelled according to the coefficients presented in table 2: $\xi_{n 1}$ (quadratic damping coefficient) represents the nonlinear damping 
term (third term in the LHS of equation 26), $c_{111}^{p}$ (nonlinear elasticity coefficient) characterizes the nonlinear restoring force (fifth term in the LHS of equation26), $e_{311}$ (nonlinear piezoelectric constant) represents the nonlinear electromechanical coupling (eighth term in the LHS of equation 26) and nonlin-geom characterizes the nonlinear geometric terms (sixth and ninth terms in the LHS of equation 26).

To better discuss the results, some contributions are presented individually and others are added to each other in Figure 8. For example, the contribution of the nonlinear damping term $\xi_{n 1}$, (blue dashed line) is presented alone. Instead, the contribution labelled by $\xi_{n 1}$ $+c_{111}^{p}$ (red dashed line) represents the combined effect of the nonlinear damping term and the nonlinear restoring force. It is interesting to observe the influence of each term to the voltage response $V(\omega)$. Regarding the nonlinear damping term, it can be clearly observed that it is not capable by itself to dampen sufficiently the response to fit the experimental data. Additionally, the nonlinear geometric terms bend the response peak to the right (typical of a hardening behavior) while the nonlinear restoring force gives a totally different (softening) response. However, compared to the experimental data, a fundamental contribution of the nonlinear electromechanical coupling appears to be necessary to match the measurements. In this sense, there is an additional damping provided by this term as can be clearly observed from Figure $8\left(\xi_{n 1}+e_{311}\right.$ green squares).

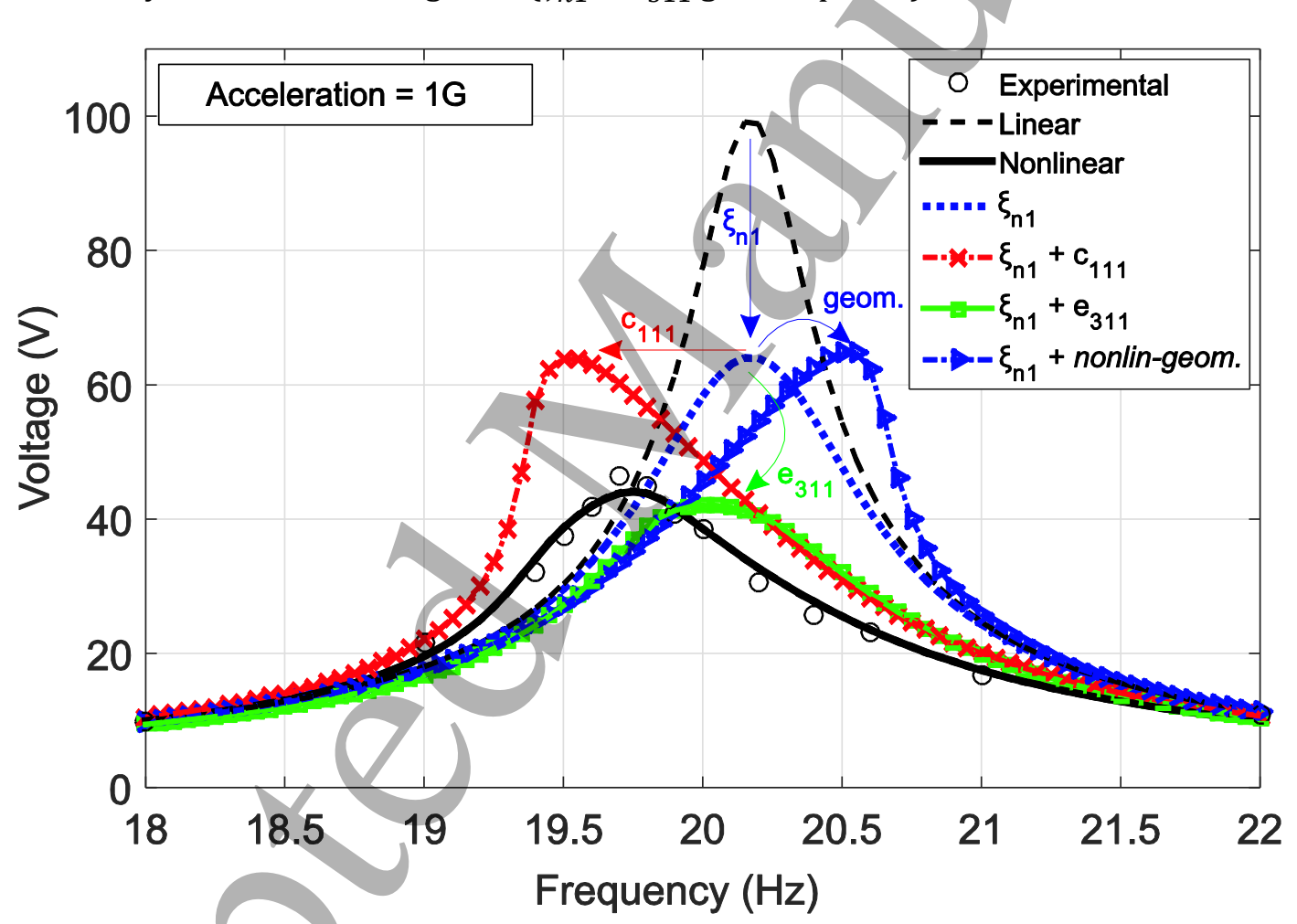

Figure 8: Contribution to the voltage of the nonlinear terms of Eqs. $26-27$ in $\mathrm{d}_{31}$ generation mode.

\subsection{MULTIMODAl DEVICE}

The last configuration tested in this section is the multimodal device developed in section 2 with a piezoelectric sheet MFC 8507-P1 (see Figure1). In this case, only numerical predictions will be compared. The interesting point inthis case is the possibility to validate 
the reduced nonlinear model for a multimodal device. To this end, the reduced model (Eqs. 32-34) with $N=3$ (red solid lines), the linear model (red dashed lines) and the direct integration of the equations of motion (Eqs. 26 and 27) with $N=3$ (blue dotted lines) are shown in Figure 6. The numerical values of the geometrical, mechanical and material parameters used in both methods are presented in Table 3.

From the analysis of the results, it is possible to conclude that the linear model always overestimates the voltage generation, especially for the first and third modes.

Considering the nonlinear model, a good general agreement between the curves is observed for the three modes. However, there are some frequency regions wherethe reduced model overestimates the voltage obtained from the direct integration values, particularly for the second and third mode.

\begin{tabular}{cccccc}
\hline Coefficient & Value & Coefficient & Value & Coefficient & Value \\
\hline$L_{1}$ & $23.3 \mathrm{~mm}$ & $a$ & $0.176 \mathrm{~mm}$ & $\xi_{n 1}$ & $1.387 \times 10^{-4}$ \\
$L_{2}$ & $85 \mathrm{~mm}$ & $\rho_{s}$ & $7900 \mathrm{~kg} / \mathrm{m}^{3}$ & $\xi_{1}$ & 0.011 \\
$L_{3}$ & $17.2 \mathrm{~mm}$ & $\rho_{p}$ & $5440 \mathrm{~kg} / \mathrm{m}^{3}$ & $\xi_{2}$ & 0.039 \\
$L$ & $125.5 \mathrm{~mm}$ & $c_{11}^{s}$ & $193 \mathrm{GPa}$ & $\xi_{3}$ & 0.008 \\
$h_{s}$ & $0.38 \mathrm{~mm}$ & $c_{11}^{p}$ & $15.85 \mathrm{GPa}$ & $\mathrm{m}_{1}$ & $19.1 \mathrm{~g}$ \\
$b_{s}$ & $12.7 \mathrm{~mm}$ & $C_{p}$ & $2.37 \mathrm{nF}$ & $m_{2}$ & $6.09 \mathrm{~g}$ \\
$h_{f}$ & $0.18 \mathrm{~mm}$ & $d_{33}$ & $440 \mathrm{pm} / \mathrm{V}$ & $k_{1}$ & $4708 \mathrm{~N} / \mathrm{m}$ \\
$b_{f}$ & $0.355 \mathrm{~mm}$ & $\epsilon_{11}^{\varepsilon}$ & $7.01 \mathrm{nF} / \mathrm{m}$ & $k_{2}$ & $3217 \mathrm{~N} / \mathrm{m}$ \\
$h_{k}$ & $0.06 \mathrm{~mm}$ & $\alpha$ & 0.2 & $k_{t 1}$ & $0.54 \mathrm{Nm} / \mathrm{rad}$ \\
$l_{p}$ & $0.41 \mathrm{~mm}$ & $\beta$ & 0.2 & $k_{t 2}$ & $0.36 \mathrm{Nm} / \mathrm{rad}$ \\
$w_{p}$ & $0.097 \mathrm{~mm}$ & $c_{111}^{p}$ & 0 & $J_{1}$ & $3.35 \times 10^{-7} \mathrm{~kg} / \mathrm{m}^{2}$ \\
$n_{f}$ & 18 & $e_{333}$ & $6.5663 \times 10^{4}$ & $J_{2}$ & $5.86 \times 10^{-8} \mathrm{~kg} / \mathrm{m}^{2}$ \\
\hline
\end{tabular}

Table 3: Material and geometrical parameters of the model.

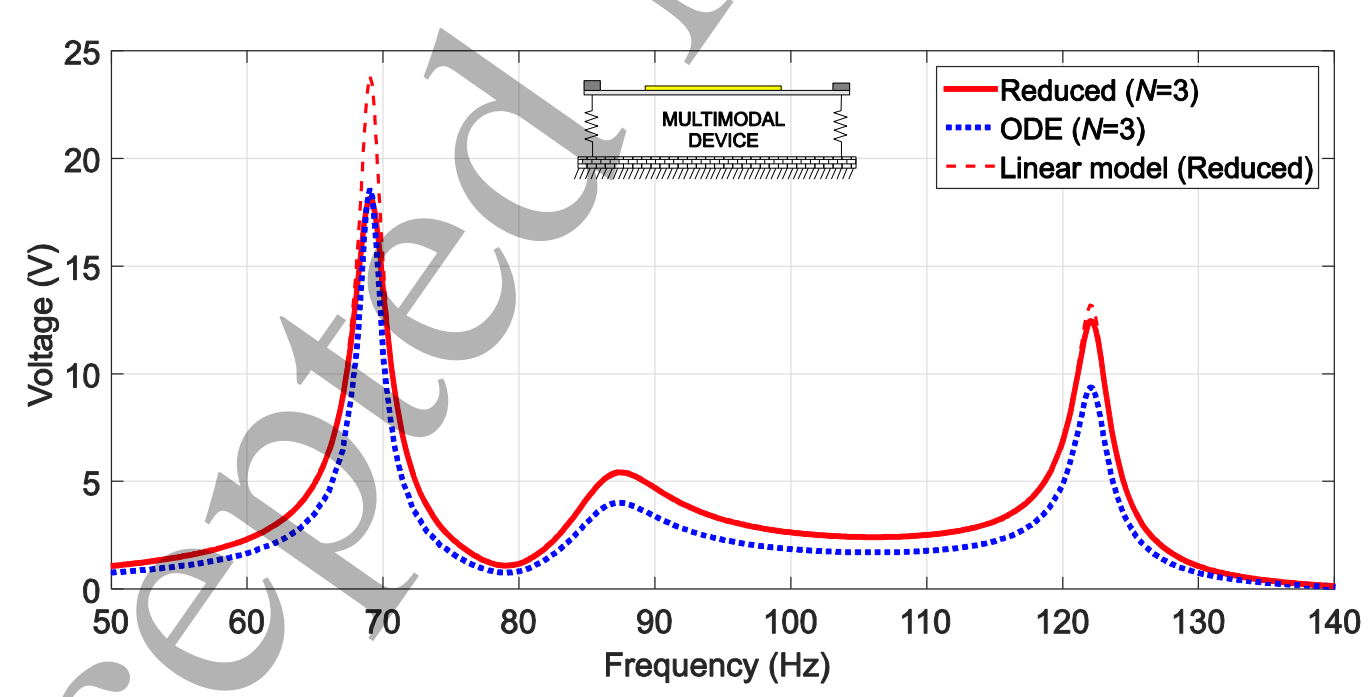

Figure9: Comparison of voltage curves for $N=3$ between the reduced model and the direct integration of the equations of motion (ODE).

Then, it is worth to analyze the sources of these deviations. If we look at section 3 , equation (28), it is possible to observe that only the first harmonic is taking into account in the reduced model. To analyze the consequences of this approach, Figure10shows the steady state of the output voltage and its spectrum (coming from the direct integration of 
the equations of motion) in a temporal interval of 0.1 seconds for two different excitation frequencies: 100 and $122 \mathrm{~Hz}$. From the figure, it is possible to observe that the steady state response seems to have a sinusoidal appearance for both analyzed cases. This is confirmed by its spectrum which is shown in the bottom part of the same figure. In this case, the contribution of the first harmonic represents almost the $90 \%$ of the total amplitude for both excitation frequencies. As a consequence, it is almost irrelevant for the present case to take higher harmonics in the approximate solution for the reduction of the system. Another source of possible discrepancy between the methods is the approximation of the sign function assumed in the approximate analytical solution provided by the reduction of the equations.

a)

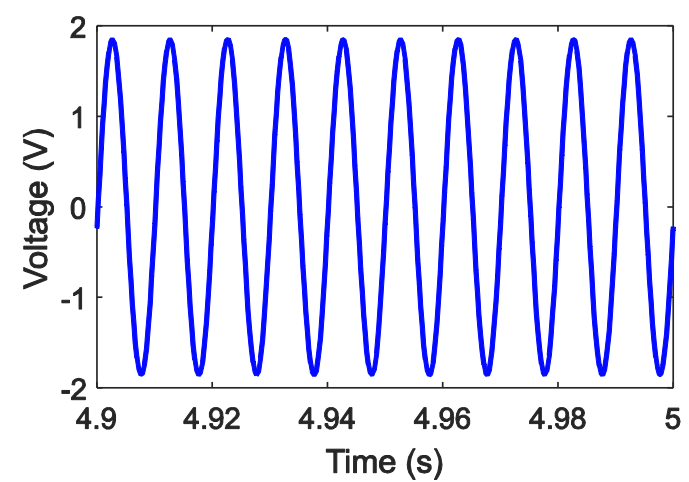

b)

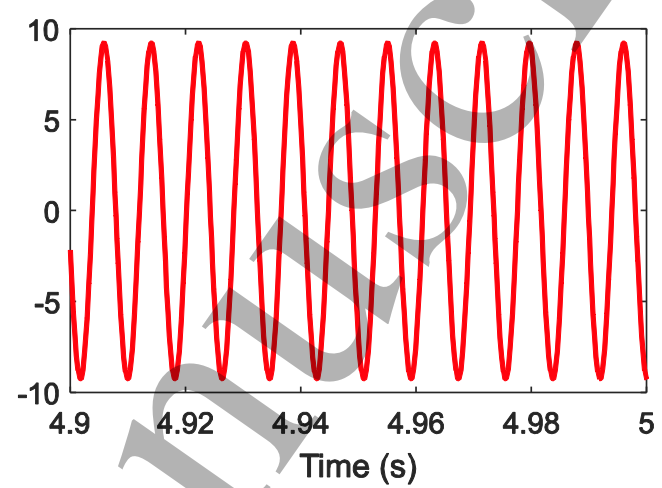

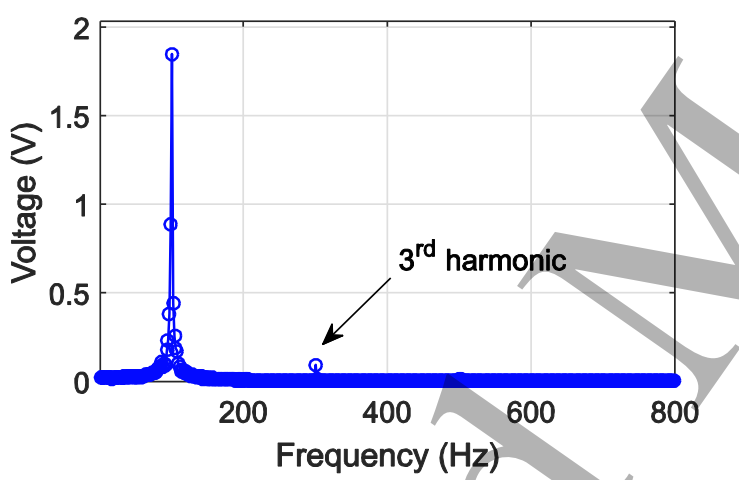

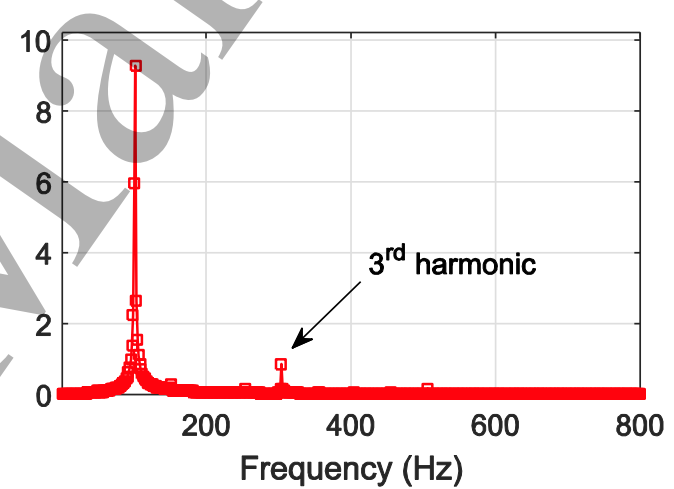

Figure10: Temporal response and spectrum at a) $100 \mathrm{~Hz}$ (off-resonance)andb) $122 \mathrm{~Hz}$ (resonance).

In order to show the importance of the reduced model, Table4 shows the computational calculation times to solve each system as a function of the number of modes. In addition, it shows the relative percentage that it takes to solve the ODES. From the times observed in the first and second columns, it can be clearly deduced that the time it takes to solve a system with $N=3$ represent $5.5 \%$ of the time tosolve the ODE. And this difference is increased when increasing the number of modes, reaching $1.7 \%$ when $N=6$.

\begin{tabular}{|c|c|c|c|}
\hline & \multicolumn{2}{|c|}{ Time (seconds) } & \multirow{2}{*}{$\begin{array}{c}\text { Reduced } \\
\text { relative to ODE }\end{array}$} \\
\hline & ODE & Reduced & \\
\hline$N=3$ & 271.11 & 15.02 & $5.54 \%$ \\
\hline$N=4$ & 515.69 & 20.69 & $4.01 \%$ \\
\hline$N=5$ & 1143.43 & 28.56 & $2.49 \%$ \\
\hline$N=6$ & 2274.13 & 38.84 & $1.70 \%$ \\
\hline
\end{tabular}

Table4: Calculation time for the reduced and ODE models 
By analyzing the difference provoked by the reduced equations it can be said that it is acceptable taking into account the tradeoff introduced by the great reduction in calculation time. This saving in time makes it possible the implementation of an identification and/or optimization process in a reasonable time with the aim of maximizing the output power of the energy harvesting device.

\section{CONCLUSIONS}

This paper presented a nonlinear mathematical formulation for inextensional BernoulliEuler beams with (unimorph or bimorph) piezoelectric sheets working in $d_{31}$ or $d_{33}$ mode that combines the most relevant contributions of the literature to this problem: (i) geometric nonlinearity induced by large displacements, (ii) material nonlinearities coming from ferroelastic hysteresis and (iii) quadratic non-linear viscous damping.

The final analytical model yields a total set of $N+1$ ordinary differential equations for the first $N$ modes and for the voltage output of the piezoelectric sheet. However, the direct solution of this ordinary nonlinear differential system of $N$ equations is computationally costly. As an alternative, a reduced algebraic system of $2(N+1)$ algebraic equations is proposed which was obtained by using the method of averaging. The main advantage of this reduced system is that it makes more suitable and computationally economical for the implementation of a parameter identification process.

Additionally, it is demonstrated that the linear formulation overestimates the generated voltage in a resonance condition and this effect is larger for a cantilever beam in a $\mathrm{d}_{31}$ generation mode. In the same sense, the contribution of each nonlinear parameter is carefully analyzed for cantilever beams, showing the importance of nonlinear analysis in this type of problems.

Several numerical and experimental tests were conducted to test the validity of the assumptions: an MFC piezoelectric cantilever beam in $d_{33}$ and $d_{31}$ mode and a multimodal system with an MFC in $d_{33}$ mode. After performing an identification process using the proposed analytical model to obtain the linear and nonlinear coefficients, the reduced algebraic system is validated against numerical and experimental data.

\section{ACKNOWLEDGMENTS}

The authors wish to thank CONICET, ANPCyT, UNS and Secretaría de Ciencia y Tecnología UTN-FRBB.

APPENDIX A: COEFFICIENTS

$$
\begin{aligned}
& M_{i}=1 \quad, \quad C_{i}=2 \xi_{i} \omega_{i}, \quad K_{i}=\omega_{i}{ }^{2} \\
& C_{n i j k} \\
& =2 \xi_{n i} \omega_{i} \int_{L_{1}}^{L_{1}+L_{2}} \phi_{i} \phi_{j} \phi_{k} \operatorname{sign}\left(\phi_{j}\right) d x \\
& K_{n i j k}=E I_{n} \int_{L_{1}}^{L_{1}+L_{2}} \phi_{i}\left(\phi_{j}{ }^{\prime \prime \prime} \phi_{k}{ }^{\prime \prime \prime}+\phi_{j}{ }^{\prime \prime} \phi_{k}{ }^{I V}\right) \operatorname{sign}\left(\phi_{j}{ }^{\prime \prime}\right) d x
\end{aligned}
$$




$$
\begin{aligned}
& K_{G i j k l}=E I \int_{0}^{L} \phi_{i}\left(\phi_{j}{ }^{\prime \prime} \phi_{k}{ }^{\prime \prime} \phi_{l}{ }^{\prime \prime}+4 \phi_{j}{ }^{\prime} \phi_{k}{ }^{\prime \prime} \phi_{l}{ }^{\prime \prime \prime}+\phi_{j}{ }^{\prime} \phi_{k}{ }^{\prime} \phi_{l}{ }^{I V}\right) d x \\
& \quad=E I_{1,3}\left(\int_{0}^{L_{1}}(\ldots) d x+\int_{L_{1}+L_{2}}^{L}(\ldots) d x\right)+E I_{2} \int_{L_{1}}^{L_{1}+L_{2}}(\ldots) d x \\
& \theta_{i}=\int_{L_{1}}^{L_{1}+L_{2}} \phi_{i} J_{p}(x)^{\prime \prime} d x \\
& \theta_{n i j}=\int_{L_{1}}^{L_{1}+L_{2}} J_{p n}(x) \phi_{i} \phi_{j}{ }^{I V} \operatorname{sign}\left(\phi_{j}{ }^{\prime \prime}\right) d x \\
& \theta_{G i j k}=\int_{L_{1}}^{L_{1}+L_{2}} \phi_{i}\left(J_{p}(x)^{\prime} \phi_{j}{ }^{\prime} \phi_{k}{ }^{\prime \prime}+\frac{1}{2} J_{p}(x)^{\prime \prime} \phi_{j}{ }^{\prime} \phi_{k}{ }^{\prime}\right) d x \\
& M_{a i}=\rho A \int_{0}^{L} \phi_{i} d x=\rho A_{1,3}\left(\int_{0}^{L_{1}} \phi_{i} d x+\int_{L_{1}+L_{2}}^{L} \phi_{i} d x\right)+\rho A_{2} \int_{L_{1}}^{L_{1}+L_{2}} \phi_{i} d x \\
& \psi_{i}=\gamma J_{p} \int_{L_{1}}^{L_{1}+L_{2}} \phi_{i}{ }^{\prime \prime} d x
\end{aligned}
$$$$
\psi_{n i j}=\gamma J_{p n} \int_{L_{1}}^{L_{1}+L_{2}} \phi_{i}{ }^{\prime \prime} \phi_{j}{ }^{\prime \prime} \operatorname{sign}\left(\phi_{j}{ }^{\prime \prime}\right) d x
$$$$
\psi_{G i j k}=\gamma J_{p} \int_{L_{1}}^{L_{1}+L_{2}}\left({\phi_{i}}^{\prime} \phi_{j}{ }^{\prime} \phi_{k}{ }^{\prime \prime}+\frac{1}{2} \phi_{j}{ }^{\prime} \phi_{k}{ }^{\prime} \phi_{i}{ }^{\prime \prime}\right) d x
$$$$
E I_{1,3}=\frac{1}{12} c_{11}^{s} b_{s} h_{s}{ }^{3}
$$$$
E I_{2}=\frac{1}{3}\left[c_{11}^{s} b_{s} h_{s}\left(3 a^{2}-3 a h_{s}+h_{s}^{2}\right)+c_{11}^{p} b_{p} h_{p}\left(3 a^{2}+3 a h_{p}+h_{p}{ }^{2}\right)\right]
$$$$
E I_{n}=\frac{1}{2} c_{111}^{p} b_{p} h_{p}\left(4 a^{3}+6 a^{2} h_{p}+4 a h_{p}^{2}+h_{p}^{3}\right)
$$$$
a=\frac{c_{11}^{s} b_{s} h_{s}^{2}-c_{11}^{p} b_{p} h_{p}^{2}}{2\left(c_{11}^{s} b_{s} h_{s}+c_{11}^{p} b_{p} h_{p}\right)}
$$$$
C_{p}=\frac{n \epsilon_{11} b_{p} h_{p}}{l_{p}}
$$$$
\rho A_{1,3}=\rho_{s} A_{s}=\rho_{s} b_{s} h_{s}
$$$$
\rho A_{2}=\rho_{s} A_{s}+\rho_{p} A_{p}=\rho_{s} b_{s} h_{s}+\rho_{p} b_{p} h_{p}
$$$$
m_{t}=\rho_{s} A_{s} L+\rho_{p} A_{p} L_{2}
$$$$
J_{p}=\frac{1}{2} e_{33} n_{f} b_{f} \frac{h_{f}}{l_{p}}\left(2 a+2 h_{k}+h_{f}\right)(1-2 \alpha)
$$ 
$J_{p n}=\frac{1}{3} e_{311} n_{f} b_{f} \frac{h_{f}}{l_{p}}\left[3 a^{2}+3 a\left(h_{f}+2 h_{k}\right)+3 h_{k}\left(h_{f}+h_{k}\right)+h_{f}^{2}\right](1-2 \alpha)$

Where $n_{f}$ is the total number of piezoelectric fibers, $b_{f}$ is the width of each fiber, and $h_{f}$ and $h_{k}$ are the thickness of the fiber and the polyamide respectively, as can be observed in Figure 11. Parameter $\alpha$ is a correction empiric parameter introduced to take into account the non-uniformity of the electric field in the thickness of the fiber [31].
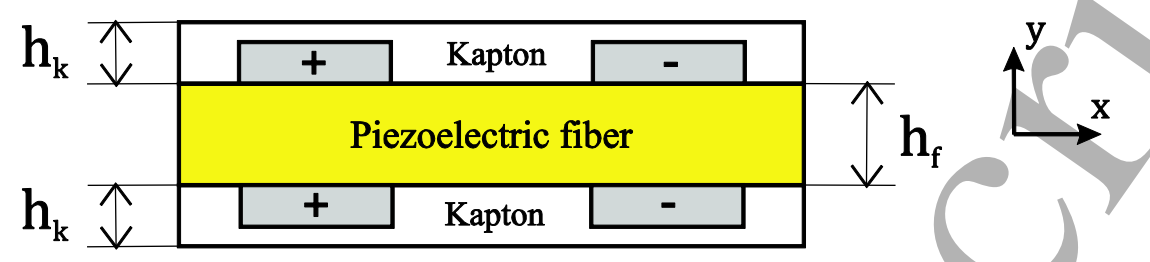

Figure11: Detail of a sheet of piezoelectric fibers of interdigitated electrodes for $\mathrm{d}_{33}$ mode generation.

For a $\mathrm{d}_{33}$ generation mode, the positions $x_{i 1}$ of function $f_{H}(x)$ related with Figure12, are given by:

$$
\text { for } i=1: \quad x_{11}=L_{1}, \quad \text { for } i=k: \quad x_{k 1}=x_{(k-1) 6}
$$

and for the rest:

$$
\begin{gathered}
x_{i 2}=x_{i 1}+\frac{w_{p}}{4}, x_{i 3}=x_{i 2}+l_{p}+\frac{w_{p}}{2} \\
x_{i 4}=x_{i 3}+\frac{w_{p}}{2}, x_{i 5}=x_{i 4}+l_{p}+\frac{w_{p}}{2}, x_{i 6}=x_{i 5}+\frac{w_{p}}{4}
\end{gathered}
$$

\section{$i^{\text {th }}$ pair of electrodes}

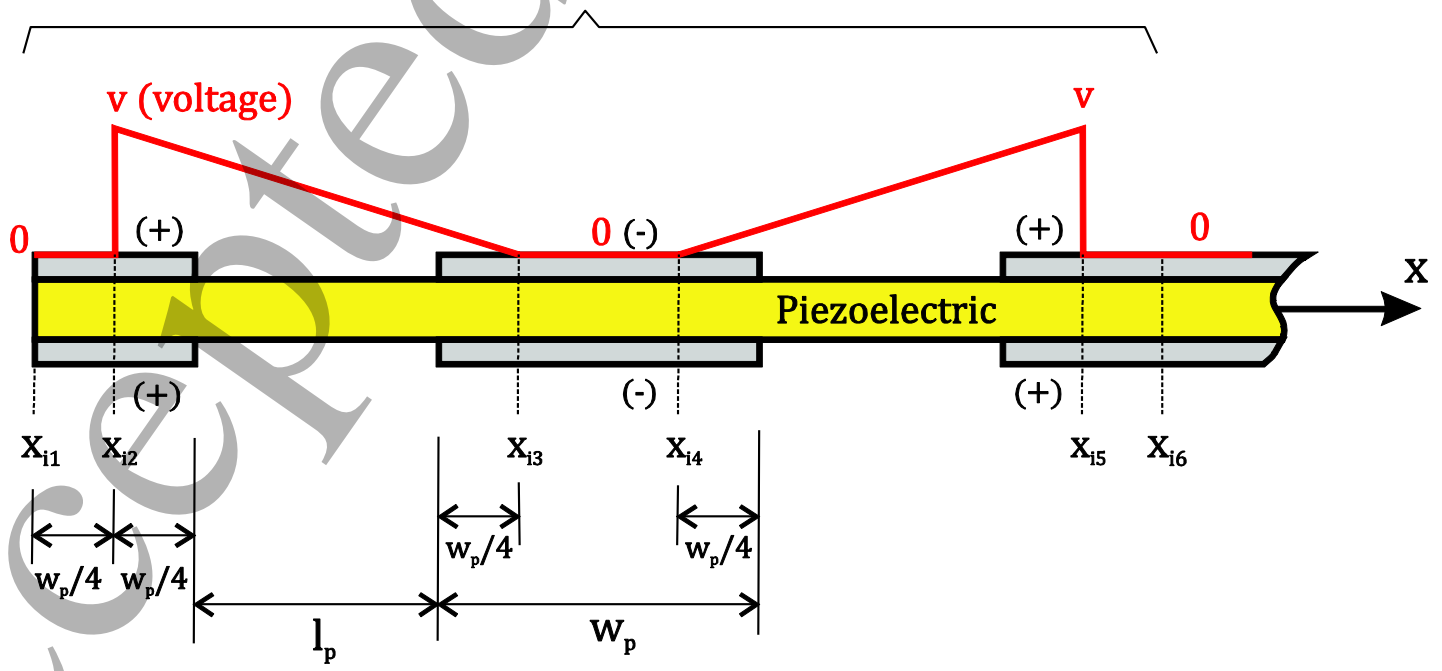

Figure12: Schematic representation of interdigitated electrodes for $\mathrm{d}_{33}$ generation mode. 
For a $\mathrm{d}_{31}$ generation mode, the detail of the geometric parameters and the polarization of the electrodes over a sheet of piezoelectric fibers is shown in Figure 13.
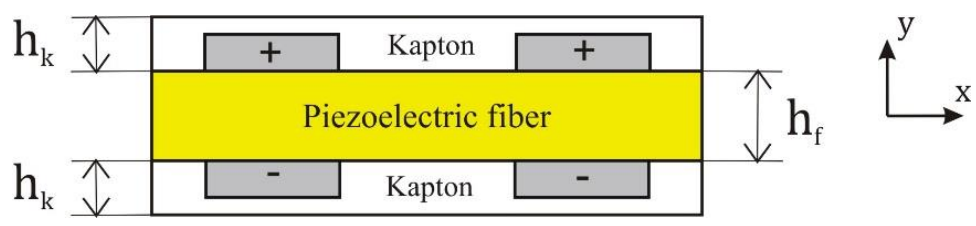

Figure13: Detail of a sheet of piezoelectric fibers of interdigitated electrodes for $\mathrm{d}_{31}$ mode generation.

The electric potential for a $d_{31}$ generation mode over the length of the MFC is shown in Figure 14.

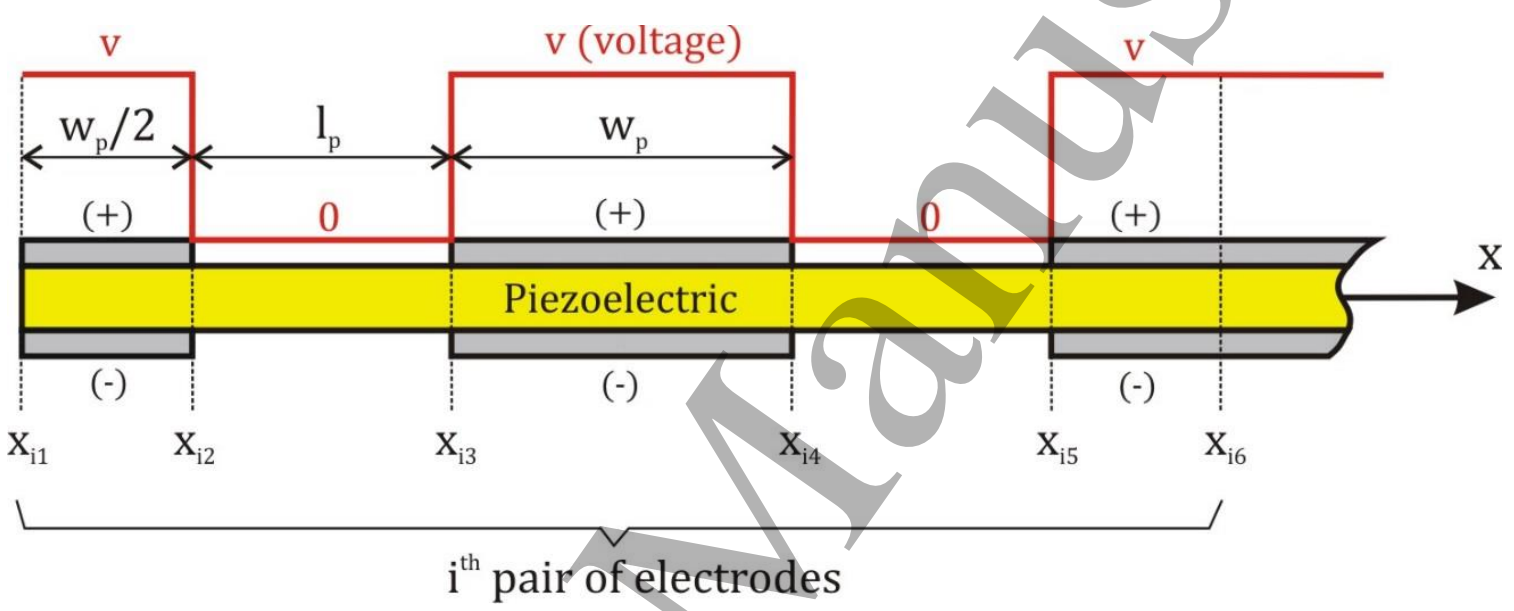

Figure 14: Schematic representation of interdigitated electrodes for $\mathrm{d}_{31}$ generation mode.

For a $\mathrm{d}_{31}$ generation mode, the positions $x_{i 1}$, of function $f_{H}(x)$ related with Figure 14 , are given by:

$$
\text { for } i=1: \quad x_{11}=L_{1}, \quad \text { for } i=k: \quad x_{k 1}=x_{(k-1) 6}
$$

and for the rest:

$$
\begin{gathered}
x_{i 2}=x_{i 1}+\frac{w_{p}}{2}, \quad x_{i 3}=x_{i 2}+l_{p} \\
x_{i 4}=x_{i 3}+w_{p}, \quad x_{i 5}=x_{i 4}+l_{p}, \quad x_{i 6}=x_{i 5}+\frac{w_{p}}{2}
\end{gathered}
$$


CONFLICT OF INTERESTS: The authors declare that they have no conflict of interest.

\section{REFERENCES}

[1] Meitzler A, Tiersten H, Warner A, Berlincourt D, Couqin G and Welsh III F 1988: Society)

[2] Gatti C D, Ramirez J M, Machado S P and Febbo M 2016 J. Phys. Conf. Ser. 773

[3] Sadeqi S, Arzanpour S and Hajikolaei K H

[4] Zhu D, Tudor M J and Beeby S P 2010 Measurement Science and Technology 21 022001

[5] Beeby S P, Tudor M J and White N 2006 Measurement science and technology 17 R175

[6] Erturk A and Inman D J 2011 Piezoelectric energy harvesting: John Wiley \& Sons)

[7] Liu M and Gorman D 1995 Computers \& structures 57 277-85

[8] Stanton S C, Erturk A, Mann B P and Inman D J 2010 Journal of Applied Physics108 074903

[9] Mak K H, Popov A A and McWilliam S 2012 Journal of Sound and Vibration331 2602-23

[10] Joshi S P 1992 Smart Mater. Struct.180

[11] Bertotti G and Mayergoyz I D 2006 The science of hysteresis: Hysteresis in materials vol 3: Gulf Professional Publishing)

[12] Damjanovic D 2006 The science of hysteresis3 337-465

[13] Goldschmidtboeing F, Eichhorn C, Wischke M, Kroener M and Woias P 2011 In: Proceedings of the 11th International Workshop on Micro and Nanotechnology for Power Generation and Energy Conversion Applications, pp 114-7

[14] Aurelle N, Guyomar D, Richard C, Gonnard P and Eyraud L 1996 Ultrasonics 34 187-91

[15] Albareda A, Gonnard P, Perrin V, Briot R and Guyomar D 2000 IEEE transactions on ultrasonics, ferroelectrics, and frequency control 47 844-53

[16] Priya S, Viehland D, Carazo A V, Ryu J and Uchino K 2001 Journal of applied physics 90 1469-79

[17] Leadenham S and Erturk A 2015 Nonlinear Dynam.79 1727-43

[18] Gatti C, Ramirez J, Febbo M and Machado S 2018 Journal of Mechanics of Materials and Structures 13 17-34

[19] Deuflhard P 2011 Newton methods for nonlinear problems: affine invariance and adaptive algorithms vol 35: Springer Science \& Business Media) 
[20] Beckert W and Kreher W S 2003 Computational Materials Science 26 36-45

[21] Nelson L, Bowen C, Stevens R, Cain M and Stewart M 2003 In: Smart Structures and Materials: International Society for Optics and Photonics pp 556-67

[22] Rao S S 2007 Vibration of continuous systems: John Wiley \& Sons)

[23] Nayfeh A H, Frank Pai P 2004 Linear and Nonlinear Structural mechanics: John Wiley \& Sons)

[24] MeIrovitch L AND PARKER R 2001 APPLIED MECHANICS REVIEWS 54100

[25] Joule J P 1847 The London, Edinburgh, and Dublin Philosophical Magazine and Journal of Science 30 76-87

[26] Bandstra J 1983 Journal of Vibration, Acoustics, Stress, and Reliability in Design 105 382-92

[27] Yang Y and Upadrashta D 2016 Nonlinear Dynam. 84 2487-504

[28] Weaver Jr W, Timoshenko S P and Young D H 1990 Vibration problems in engineering: John Wiley \& Sons)

[29] Stewartson K 1966: Wiley Online Library)

[30] Dormand J R and Prince P J 1980 Journal of computational and applied mathematics $619-26$

[31] Bilgen O, Erturk A and Inman D 2010 Journal of Vibration and Acoustics 132 051005 